INSTITUT NATIONAL DE RECHERCHE EN INFORMATIQUE ET EN AUTOMATIQUE

\title{
Energy-aware mappings of series-parallel workflows onto chip multiprocessors
}

\author{
Anne Benoit — Rami Melhem — Paul Renaud-Goud — Yves Robert
}

\section{$\mathbf{N}^{\circ} \mathbf{7 5 2 1}$}

January 2011

Distributed and High Performance Computing

apport

de recherche 



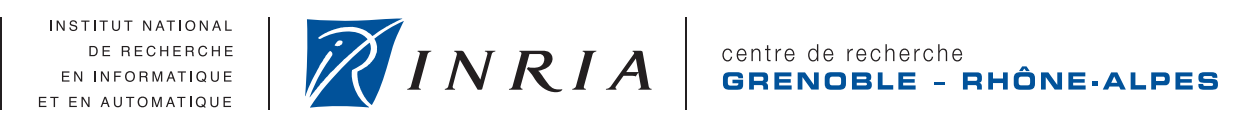

\title{
Energy-aware mappings of series-parallel workflows onto chip multiprocessors
}

\author{
Anne Benoit , Rami Melhem, Paul Renaud-Goud, Yves Robert \\ Theme : Distributed and High Performance Computing \\ Équipe-Projet GRAAL
}

Rapport de recherche $\mathrm{n}^{\circ} 7521$ - January $2011-34$ pages

\begin{abstract}
This paper studies the problem of mapping streaming applications that can be modeled by a series-parallel graph, onto a 2-dimensional tiled CMP architecture. The objective of the mapping is to minimize the energy consumption, using dynamic and voltage scaling techniques, while maintaining a given level of performance, reflected by the rate of processing the data streams. This mapping problem turns out to be NP-hard, but we identify simpler instances, whose optimal solution can be computed by a dynamic programming algorithm in polynomial time. Several heuristics are proposed to tackle the general problem, building upon the theoretical results. Finally, we assess the performance of the heuristics through comprehensive simulations using the StreamIt workflow suite and various CMP grid sizes.
\end{abstract}

Key-words: series-parallel graph; DAG; mapping; multicore; CMP; energy; power; period; throughput; DVS; DVFS; complexity; simulation; streaming applications; optimization. 


\section{Energy-aware mappings of series-parallel workflows onto chip multiprocessors}

Résumé : Dans ce rapport de recherche, nous nous intéressons au placement d'applications de type streaming représentées sous la forme d'un graphe sérieparallèle sur un processeur multi-cœur, en essayant de minimiser l'énergie consommée tout en n'excédant pas une borne sur un critère de performance, la période. La partie théorique démontre la NP-complétude ou la polynomialité du problème, selon des propriétés structurelles du multi-cœur (chaîne de cœurs, uni- ou bi-directionnelle, grille de coeurs) et la largeur du graphe de l'application (bornée ou non). Le problème le moins contraint étant NP-complet, nous décrivons dans la partie expérimentale quatre heuristiques, puis les comparons entre elles, et donnons un programme linéaire en nombres entiers qui permet d'obtenir la solution optimale en temps exponentiel.

Mots-clés : graphe serie-parallèle; DAG; mapping; multi-cœur; énergie; puissance; période; DVS; DVFS; complexité; simulation; streaming; optimisation. 


\section{Contents}

1 Introduction $\quad 4$

2 Related work 5

3 Framework $\quad 7$

3.1 Applicative framework . . . . . . . . . . . . . . . 7

3.2 Platform . . . . . . . . . . . . . . . . . . . . 10

3.3 Mapping strategies . . . . . . . . . . . . . . . . . 10

3.4 Period ............................ 11

3.5 Energy model . . . . . . . . . . . . . . . . . 11

4 Complexity results 12

4.1 Uni-directional uni-line CMP . . . . . . . . . . . . . . . . 13

4.2 Bi-directional uni-line CMP . . . . . . . . . . . . . . . . . . . . . . . . . . . . 14

4.3 Square CMP ... . . . . . . . . . . . . . . . . . 16

4.4 Integer linear program . . . . . . . . . . . . . . . . . . . . . . . . . . . . . . . . . 19

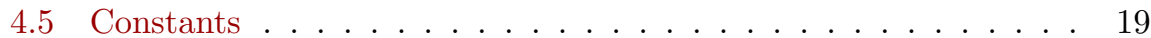

4.6 Variables . . . . . . . . . . . . . . . . . . 20

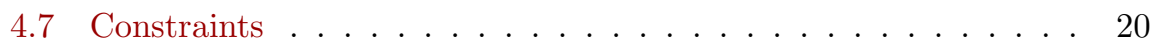

4.8 Objective function . . . . . . . . . . . . . . 22

5 Heuristics $\quad 22$

5.1 Random heuristic . . . . . . . . . . . . . . . . . . . . 22

5.2 Greedy heuristic . . . . . . . . . . . . . . . . . . . 23

5.32 D dynamic programming algorithm . . . . . . . . . . . . . 23

5.4 1D dynamic programming algorithm . . . . . . . . . . 25

6 Experiments $\quad \mathbf{2 5}$

6.1 Experimental setting . . . . . . . . . . . . . . . . . 25

6.2 Experimental results . . . . . . . . . . . . . . 27

7 Conclusion $\quad 30$ 


\section{Introduction}

The energy consumption of computational platforms has recently become a critical problem, both for economic and environmental reasons [34]. To help reduce energy consumption, processors can run at different speeds. Faster speeds allow for a faster execution, but they also lead to a much higher (superlinear) power consumption. Energy-aware scheduling aims at minimizing the energy consumed during the execution of the target application, both for computations and for communications. Obviously, this approach makes sense only if coupled with some performance bound to achieve. Otherwise, the optimal solution always is to run each resource at the slowest possible speed. In other words, we have a bi-criteria optimization problem, with one objective being energy minimization, and the other being performance-related.

In this paper, we aim at minimizing the energy consumption of streaming applications whose task graph is a series-parallel graph (SPG). Streaming applications, or workflows, are ubiquitous in many domains, as for instance DSP or image processing applications, astrophysics, meteorology, neuroscience, and so on [16, 44, 43, 52]. Most of these applications have simple and regular task graphs, such as linear chains, trees, fork-join graphs, or general SPGs (see Section 3.1 for a formal definition of SPGs.) For instance, all the benchmarks of the StreamIt suite [45] are SPGs.

The performance-related objective coupled with energy minimization is the period of the streaming application. Typically, a series of data sets enter the input stage and progress from stage to stage, following the dependencies of the application, until the final result is computed. Each stage has its own communication and computation requirements: it reads input from the previous stage(s), processes the data and outputs results to the next stage(s). The pipeline operates in a dataflow mode: after a transient behavior due to the initialization delay, a new data set is completed every period. The period, which corresponds to the inverse of the throughput, is a key performance-related objective for streaming applications [46, 16, 20]. Formally, the period is the time interval between the arrival of two consecutive data sets in the application. Given a mapping of the application onto a platform, the cycle-time of each resource (processor or communication link) should not exceed the period.

Finally, the target platform for this study is a Chip MultiProcessor (CMP), which is composed of $p \times q$ homogeneous cores arranged along a $2 \mathrm{D}$ grid. During the last century, advances in integrated circuit technology have led chip designers to increase microprocessor performance by increasing the integration density thus allowing for higher clock rates and new innovation in micro-architecture. Such innovations included wider instruction, speculative execution, branch prediction and dynamic scheduling. However, in 1996, Olukotum et al. [38] argued that such a trend would not continue because of the diminishing return caused by the limited amounts of instruction level parallelism and they argued that a better way for using the denser integration would be to layout multiple simpler processors on the same chip. Moreover, power consumption consideration prevented the push towards faster clocks, thus leaving the design of chip multiprocessors as the only alternative for increasing the on-chip computational capability. Specifically, increasing the number of cores rather than the processor's complexity translates into slower growth in power consumption. Currently, chip multiprocessors are commercially available and the trend is towards the contin- 
uous increase in the number of cores on single chips. The challenge is now to be able to efficiently utilize the parallelism available on chip [8].

An essential step for exploring the parallelism available in a streaming application is to provide algorithms and scheduling strategies for mapping a seriesparallel graph onto a CMP, with the objective of minimizing the energy consumption while not exceeding a prescribed period. In some applications, data sets arrive at fixed time intervals, and hence the period of the application is given a priori, before any mapping is computed. In other applications, there is the freedom to choose between a set of possible periods, which are prescribed by the user. In all cases, the main goal is to reduce the energy consumption of the mapping, while enforcing the constraint on the prescribed period.

The contribution of this paper is twofold. On the theoretical side, we assess the complexity of the above-mentioned mapping problem, using a general mapping rule that partitions the application SPG into an acyclic graph of node clusters. In turn, each cluster is mapped onto a different processor of the CMP. Our cost model accounts for communication delays and cost (in terms of consumed energy). The problem turns out to be NP-hard, so we also study the complexity of simpler problem instances, either with a simpler target platform (uni-directional or bi-directional uni-line CMP), or by restricting to particular applications whose graph has a bounded degree of parallelism (bounded-elevation SPGs). The only problem instance that can be solved in polynomial time, thanks to a dynamic programming algorithm, is the mapping of bounded-elevation SPGs onto a uni-directional uni-line CMP. For other problem instances, we provide sophisticated NP-completeness proofs. On the practical side, we design some polynomial-time heuristics to solve the most general problem, building upon the theoretical results, and we assess their performance through a set of simulations.

The paper is organized as follows. We first survey related work in Section 2. Then we detail the framework in Section 3, and we provide complexity results in Section 4. The heuristics are described in Section 5, and experimental results in Section 6. Finally, we conclude and discuss future research directions in Section 7.

\section{Related work}

Reducing the energy consumption of computational platforms is an important research topic, and many techniques at the process, circuit design, and microarchitectural levels have been proposed [31, 29, 19]. The dynamic voltage and frequency scaling (DVFS) technique has been extensively studied, since it may lead to efficient energy/performance trade-offs [26, 18, 4, 12, 28, 51, 48]. Current microprocessors (for instance, from AMD [2] and Intel [35]) allow the speed to be set dynamically. Indeed, by lowering supply voltage, hence processor clock frequency, it is possible to achieve important reductions in power consumption, without necessarily increasing the execution time.

In this paper, we aim at minimizing the energy consumption for seriesparallel graph (SPG) applications which are mapped onto a chip multiprocessor (CMP). We first discuss related work on SPG applications, then we review different energy minimization approaches. Finally, we relate work on mapping problems on CMPs. 
Series-parallel workflow applications. Classical workflow applications usually consists of a directed acyclic graph: the application is made of several tasks, and there are dependencies between these tasks. However, it turns out that many of these graphs are series-parallel graphs. For instance, in [33], McClatchey et al. introduce a prototype scientific workflow management system entitled CRISTAL, and the distributed scientific workflow applications that they consider are SPGs. In [41], Qin and Fahringer discuss several scientific grid workflow applications, which are all structured as SPGs: the WIEN2k workflow performs electronic structure calculations of solids using density functional theory [7], the MeteoAG workflow is a meteorology simulation application [43], and the GRASIL workflow calculates the spectral energy distribution of galaxies [44]; this latter application has actually a fork-join graph. A last example is the fMRI workflow [52], which is a cognitive neuroscience application.

DVFS and optimization problems. When dealing with energy consumption, the most usual optimization function consists of minimizing the energy consumption, while ensuring some performance guarantees (real-time constraints, such as a bound on the total execution time, or a threshold throughput). Specifically, in [37], Okuma et al. demonstrate that voltage scaling is far more effective than the shutdown approach, which simply stops the power supply when the system is inactive. De Langen and Juurlink [30] discuss leakage-aware scheduling heuristics which investigate both DVS and processor shutdown, since static power consumption due to leakage current is expected to increase significantly. In the context of real-time embedded systems, Lee and Sakurai [31] show how to exploit slack time arising from workload variation, thanks to a software feedback control of supply voltage. Prathipati [40] discusses techniques to take advantage of run-time variations in the execution time of tasks; it determines the minimum voltage under which each task can be executed, while guaranteeing the deadlines of each task. In [50], dynamic programming algorithms are given to minimize the expected energy consumption in real time systems using frequency and voltage scaling. Yang and Lin [51] discuss algorithms with preemption, using DVS techniques; substantial energy can be saved using these algorithms, which succeed to claim the static and dynamic slack time, with little overhead. Most of these papers deal with classical scheduling of task graph applications, which are not streaming applications. The techniques are similar, but the performance guarantee is a deadline on the total execution time. Rather, we consider workflow applications, i.e., several data sets must be processed by the task graph, and hence we bound the application period.

The problem of mapping workflow applications with the structure of a linear chain onto parallel platforms has already been widely studied, in particular on homogeneous platforms (see the pioneering paper [47]) and later for heterogeneous platforms [6]. These results are extended to account for energy consumption in [5], where the target problem is mapping several linear chain applications on a fully interconnected platform, with three optimization criteria: power, period, and latency (execution time for one data set).

Mapping applications to chip multiprocessors. Many researchers have considered the mapping of tasks and threads to CMPs that are connected by a 2-dimentional network on a chip. The work in [3] introduces an approach to multi-objective exploration of mapping general task graphs to mesh-based CMPs using evolutionary algorithms. The approach is an efficient and accurate way to 
obtain the Pareto mappings that optimize performance and power consumption. In [25], an architecture-aware analytic mapping algorithm is presented. It uses a metric space that exactly captures the CMP topology to efficiently solve the problem. In [10], a compiler framework is presented to map the source code of an application to a mesh-based chip multiprocessor system. Compiler techniques are also used in [9] to dynamically change the speed of communication channels in CMPs to reduce energy consumption. In [1], the mapping of applications to heterogeneous multi-processor systems is performed by invoking runtime agents that are distributed among the processors. None of the above work considers the mapping of streaming applications onto CMP with the objective of minimizing power consumption while maintaining a specified throughput (period).

Summary. In this paper, the application is a workflow whose structure is series-parallel task graph, and the goal is to map this application onto a CMP, with the objective of minimizing the energy consumption, given a threshold on the period of the workflow. We are therefore extending previous work, which was conducted for simpler application structures (linear chains instead of seriesparallel graphs), and for a realistic platform (the CMP) instead of virtual cliques. To the best of our knowledge, this paper is the first to investigate the complexity of this problem, and to propose practical solutions (polynomial time heuristics) for applications modeled by series-parallel graphs. The work in [49] shares the same objective as the work in this paper but is purely empirical. It presents a two-phase heuristic for mapping a general acyclic graph onto a CMP by first assigning the levels of the graph to the rows of the CMP and then mapping the tasks in each level to the nodes of the row assigned to that level. The heuristic described in Section 5.3 follows a similar two-phase strategy.

\section{Framework}

In this section, we first describe the applicative framework (Section 3.1) and the target platform (Section 3.2). Then we detail the mapping strategies in Section 3.3. Finally, we formally define the bi-criteria optimization problem: we aim at minimizing the energy consumption while not exceeding a prescribed period. The formula to check that the period is not exceeded is given in Section 3.4, and the model for energy consumption is outlined in Section 3.5.

\subsection{Applicative framework}

The application that is to be scheduled is a streaming application: it operates on a collection of data sets that are executed in a pipelined fashion. In this study, the application is a series-parallel graph $\mathcal{G}=(\mathcal{S}, \mathcal{E})$, or SPG. Nodes of the graph correspond to different application stages, and are denoted by $S_{i}$, with $1 \leq i \leq n$, where $n=|\mathcal{S}|$ is the size of the graph. For each precedence constraint in the application, say from stage $S_{i}$ to stage $S_{j}$, we have an edge $L_{i, j} \in \mathcal{E}$. For $1 \leq i \leq n, w_{i}$ is the computation requirement of stage $S_{i}$, and for each $L_{i, j} \in \mathcal{E}$, with $1 \leq i, j \leq n, \delta_{i, j}$ is the volume of communication to be sent from $S_{i}$ to $S_{j}$ before $S_{j}$ can start its computation.

A SPG is built from a sequence of compositions (parallel or series) of smallersize SPGs. The smallest SPG consists of two nodes connected by an edge. The first node is the source, while the second one is the sink of the SPG. When 
composing two SPGs in series, we merge the sink of the first SPG with the source of the second one. For a parallel composition, the two sources are merged, as well as the two sinks (see Figure 1 for illustrative examples).

We recursively define the label of each node in a SPG, which corresponds to its coordinates along a $2 \mathrm{D}$-grid in the recursive construction: $\ell_{i}=\left(x_{i}, y_{i}\right)$ is the label of stage $S_{i}$, for $1 \leq i \leq n$. First, for a two-node SPG $\left(S_{1} \rightarrow S_{2}\right)$, the label of the source $S_{1}$ is $(1,1)$, while the label of the $\operatorname{sink} S_{2}$ is $(2,1)$. The labels are then updated when composing the SPG. Consider two SPGs, $S P G_{1}$ with nodes $S_{1}^{(1)}, \ldots, S_{n_{1}}^{(1)}$, and $S P G_{2}$ with nodes $S_{1}^{(2)}, \ldots, S_{n_{2}}^{(2)}$, and their corresponding labels $\ell_{i}^{(1)}=\left(x_{i}^{(1)}, y_{i}^{(1)}\right)$ and $\ell_{j}^{(2)}=\left(x_{j}^{(2)}, y_{j}^{(2)}\right)$, for $1 \leq i \leq n_{1}$ and $1 \leq j \leq n_{2}$.

- For a serial composition, we merge the sink of $S P G_{1}, S_{n_{1}}^{(1)}$, with the source of $S P G_{2}, S_{1}^{(2)}$. The resulting SPG has $n=n_{1}+n_{2}-1$ nodes with the following labels: for $1 \leq i \leq n_{1}, S_{i}=S_{i}^{(1)}$ and its label is $\ell_{i}=\ell_{i}^{(1)}$, and for $1<j \leq n_{2}, S_{n_{1}+j-1}=S_{j}^{(2)}$ and the $x$ values of the labels are incremented by $x_{n_{1}}^{(1)}-1$, i.e., $\ell_{n_{1}+j-1}=\left(x_{j}^{(2)}+x_{n_{1}}^{(1)}-1, y_{j}^{(2)}\right)$.

- For a parallel composition, assume that $x_{n_{1}}^{(1)} \geq x_{n_{2}}^{(2)}$ (otherwise exchange both SPGs, so that the first one contains the longest path). We merge both sources $\left(S_{1}^{(1)}\right.$ and $\left.S_{1}^{(2)}\right)$, and both sinks $\left(S_{n_{1}}^{(1)}\right.$ and $\left.S_{n_{2}}^{(2)}\right)$. The resulting SPG has $n=n_{1}+n_{2}-2$ nodes with the following labels: $S_{1}$ is the source and $\ell_{1}=\ell_{1}^{(1)} ; S_{n}$ is the sink and $\ell_{n}=\ell_{n_{1}}^{(1)}$; for $1<i<n_{1}, S_{i}=S_{i}^{(1)}$ and its label is $\ell_{i}=\ell_{i}^{(1)}$; for $1<j<n_{2}, S_{n_{1}+j-2}=S_{j}^{(2)}$, and the $y$ values of the labels are incremented by $y_{\max }^{(1)}=\max _{1 \leq i \leq n_{1}}\left(y_{i}^{(1)}\right)$, i.e., $\ell_{n_{1}+j-2}=\left(x_{j}^{(2)}, y_{j}^{(2)}+y_{\max }^{(1)}\right)$.

This construction is illustrated on the examples given in Figure 1. Note that these rules ensure that the source is always stage $S_{1}$, with $\ell_{1}=(1,1)$, and the sink is always stage $S_{n}$, with $\ell_{n}=\left(x_{n}, 1\right)$. Therefore, $\max _{1 \leq i \leq n} x_{i}=x_{n}$, and we denote by $y_{\max }=\max _{1 \leq i \leq n} y_{i}$ the maximum $y$ value of the labels in the SPG, which we call maximum elevation. Intuitively, the maximum elevation denotes the maximal degree of parallelism of the SPG.

In the following, we focus the discussion on bounded-elevation SPGs, i.e., SPGs whose maximum elevation $y_{\max }$ is bounded by a constant. Indeed, dealing with bounded-elevation SPGs, rather than arbitrary SPGs, or even arbitrary DAGs, is a trade-off between tractability and generality. On the one hand, bounded-elevation SPGs correspond to a wide spectrum of applications, and nicely generalize linear chains and trees (a tree can easily be transformed into a SPG by adding fake nodes mirroring the tree). For instance, all the benchmarks of the StreamIt suite [45] are bounded-elevation SPGs: their maximum elevation ranges from $y_{\max }=1$ (linear chain) to $y_{\max }=17$. On the other hand, the problem of mapping a simple fork-join graph with $n$ nodes (unbounded-elevation graph) onto two processors, in order to minimize the energy given a period bound, is NP-complete (reduction from 2-PARTITION, see Section 4.1). Dealing with bounded-elevation graphs enables us to identify polynomial instances, hence to provide optimal solutions, for some problem instances. 

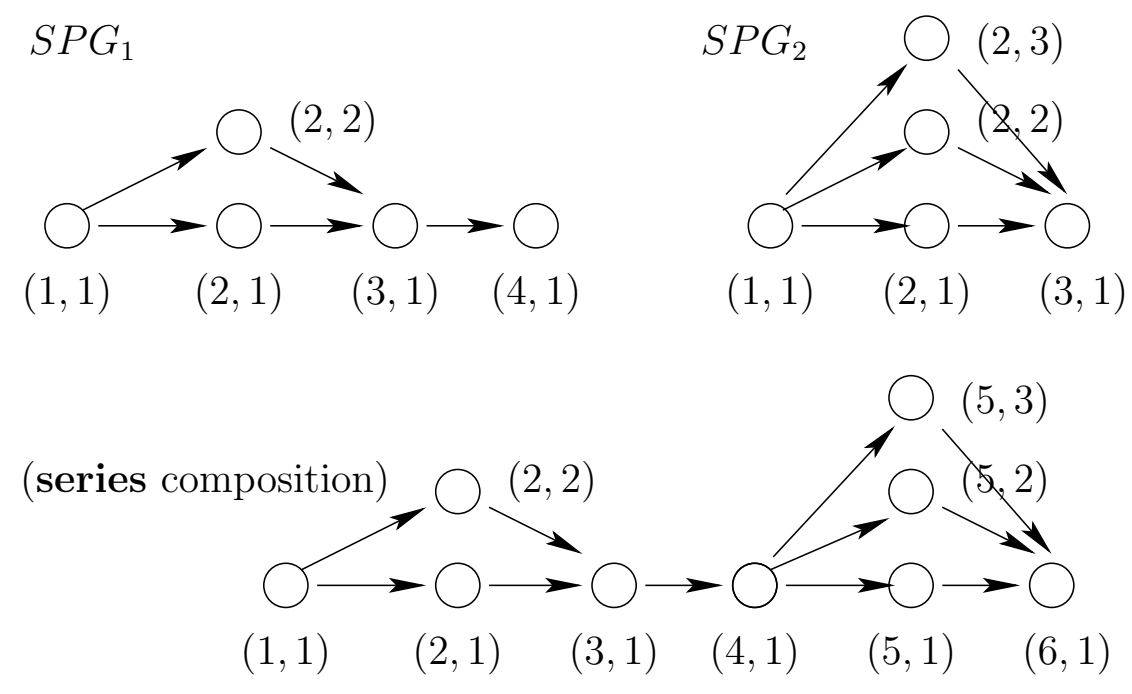

(parallel composition)

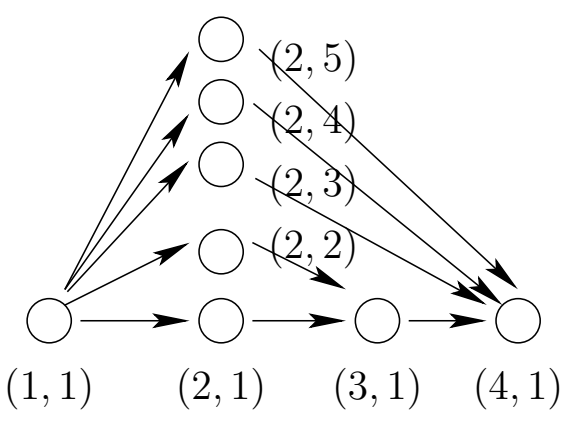

Figure 1: Examples of SPG composition. 


\subsection{Platform}

The target platform is a CMP (Chip MultiProcessor), composed of $p \times q$ homogeneous cores $\mathcal{C}_{u, v}$, with $1 \leq u \leq p, 1 \leq v \leq q$, arranged along a rectangular matrix. There is a vertical (internal and bi-directional) communication link between $\mathcal{C}_{u, v}$ and $\mathcal{C}_{u+1, v}$, for $1 \leq u \leq p-1,1 \leq v \leq q$, and a horizontal link between $\mathcal{C}_{u, v}$ and $\mathcal{C}_{u, v+1}$, for $1 \leq u \leq p, 1 \leq v \leq q-1$. All links have the same bandwidth $B W$ (in each direction). This means that it takes a time $\frac{\delta}{B W}$ to send $\delta$ bytes from one processor to another neighboring processor. It is possible to use only some of the communication links, and for instance to configure the $p \times q \mathrm{CMP}$ as a $1 \times p q$ bi-directional linear array, called bi-directional uni-line $C M P$.

Although the cores of a CMP share the same memory space, it is possible to implement the message passing models on CMPs [32] by writing and reading from shared memory locations. However, for scalability purpose, CMPs with large number of cores will be organized as a mesh of tiles, each with its own cache [27]. Therefore, communication through shared memory ultimately translates to exchange of coherence traffic between the tiles [22, 14, 24]. Specifically, in the streaming model assumed in this paper, when a stage $S_{i}$, mapped to a core $\mathcal{C}_{u, v}$, writes into a shared variable, $X$, that shared variable is cached in the local cache of $\mathcal{C}_{u, v}$. Then, when a stage $S_{j}$ with $L_{i, j} \in \mathcal{E}$, mapped to a core $\mathcal{C}_{u^{\prime}, v^{\prime}} \neq \mathcal{C}_{u, v}$, reads $X$, the cache coherence protocol guarantees that $X$ is cached in the local cache of $\mathcal{C}_{u^{\prime}, v^{\prime}}$. Therefore, the values of the cache line containing $X$ have been sent from $\mathcal{C}_{u, v}$ to $\mathcal{C}_{u^{\prime}, v^{\prime}}$. In other words, if two stages $S_{i}$ and $S_{j}$, connected with an edge $L_{i, j}$, are mapped onto two distinct processors, a communication of size $\delta_{i, j}$ must occur (implicit messages) to keep the cached values coherent ${ }^{1}$. Hence, irrespectively of the programming model used to implement the SPG, the weight on a directed edge between two nodes in the SPG represents the volume of communication to be sent between the cores executing the corresponding application stages.

As mentioned in Section 2, the voltage and frequency of each core of the $\mathrm{CMP}$ can be set to different values. Altogether, there is a set of possible supply voltages, together with a set of possible frequencies (or modes, or speeds), for each core. Let $\mathrm{S}=\left\{s^{(1)}, \ldots, s^{(m)}\right\}$ denote the set of all possible speeds. It takes a time $\frac{w_{i}}{s^{(k)}}$ to execute one data set for stage $S_{i}$ at speed $s^{(k)} \in \mathrm{S}$ on a given core. Each speed induces a different dynamic power consumption, as discussed in Section 3.5 below.

\subsection{Mapping strategies}

We discuss several mapping rules to map the SPG application onto the CMP. As for the application graph, we use DAG-partition mappings, which represent a trade-off between one-to-one and general mappings. The rationale is the following. One-to-one mappings obey the simplest rule: each application stage is mapped onto a distinct core. While easier to optimize and implement, this rule may be unduly restrictive, and is likely to lead to high communication costs. Obviously, it also requires that $p \times q \geq n$, thereby limiting its applicability to large platforms or small applications. A natural extension is to search for

\footnotetext{
${ }^{1}$ It is assumed that the cache coherence protocol is capable of exploiting communication locality by tracking in each core the location of frequently accessed blocks [21].
} 
DAG-partition mappings: we first partition the initial SPG into subsets, or clusters, such that the resulting graph is acyclic. Hence this mapping rule states that if two stages $S_{i}$ and $S_{j}$ are in the same subset of the partition, then any other stage $S_{k}$ which has an incoming dependency from $S_{i}$ and an outgoing dependency to $S_{j}$, must be in the same subset of the partition. Then we map the subsets of the partition onto the cores in a one-to-one fashion. Using this mapping rule, a core which is executing a subset $I$ of stages $\left\{S_{i}\right\}_{i \in I}$ will perform at most one input and one output communication for each elevation value $\left\{y_{i}\right\}_{i \in I}$. This is well in accordance with our initial assumption that the SPG has bounded elevation $y_{\max }$, because it ensures that each core has at most $y_{\max }$ communications to perform at each period. In contrast, a fully general mapping, that allow for arbitrary partitions of the original application graph, would require an arbitrary number of communications, only bounded by the total number of stages $n$, hence an unlimited amount of buffer space. Moreover, even for bounded-elevation SPGs, the problem of finding the general mapping which minimizes the energy given a period bound is trivially NP-complete (linear chain onto two processors, reduction from 2-PARTITION [17]).

Formally, the mapping is defined by an allocation function

$$
\text { alloc }:\{1, \ldots, n\} \rightarrow\{1, \ldots, p\} \times\{1, \ldots, q\},
$$

which maps stages onto cores. In other words, if stage $S_{i}$ is mapped onto core $\mathcal{C}_{u, v}$, we have $\operatorname{alloc}(i)=(u, v)$. Once application stages are mapped onto cores, there remains to decide how to route communications between two cores which need to communicate because of the stage assignment. Therefore, for each application edge $L_{i, j} \in \mathcal{E}$, if $\operatorname{alloc}(i) \neq \operatorname{alloc}(j)$, we define $\operatorname{path}_{i, j}$ as the set of communication links that are used to communicate from core alloc $(i)$ to core alloc $(j)$. Note that these paths must be defined for the mapping to be fully determined.

\subsection{Period}

As motivated above, we assume that data sets arrive at regular time intervals, which is called the period of the application, and denoted by $T$. Then, given a mapping and an execution speed for each core, we can check whether the application can be executed at the prescribed rate: we must ensure that the cycle-time of each resource (computation or communication link) does not exceed $T$.

Let $w_{u, v}=\sum_{1 \leq i \leq n \mid \operatorname{alloc}(i)=(u, v)} w_{i}$ be the total amount of work assigned to core $\mathcal{C}_{u, v}$, running at speed $s_{u, v} \in \mathrm{S}$. The cycle-time of $\mathcal{C}_{u, v}$ for computations is $w_{u, v} / s_{u, v}$. For communications, $b_{(u, v) \rightarrow\left(u^{\prime}, v^{\prime}\right)}=\sum_{1 \leq i, j \leq n \mid(u, v) \rightarrow\left(u^{\prime}, v^{\prime}\right) \in \text { path }_{i, j}} \delta_{i, j}$ is the number of bits sent from $\mathcal{C}_{u, v}$ to a neighbor core $\mathcal{C}_{u^{\prime}, v^{\prime}}{ }^{2}$. The cycle-time of the communication link $(u, v) \rightarrow\left(u^{\prime}, v^{\prime}\right)$ is $b_{(u, v) \rightarrow\left(u^{\prime}, v^{\prime}\right)} / B W$.

We can then compute the maximum cycle-time, which is the maximum cycletime of all resources, and check that it is not greater than $T$.

\subsection{Energy model}

Once a SPG application has been mapped onto the CMP, there are two sources of energy consumption: on the one hand, the cores consume energy for com-

\footnotetext{
${ }^{2}\left(u^{\prime}=u+1\right.$ and $\left.v^{\prime}=v\right)$ or $\left(u^{\prime}=u-1\right.$ and $\left.v^{\prime}=v\right)$ or $\left(u^{\prime}=u\right.$ and $\left.v^{\prime}=v+1\right)$ or $\left(u^{\prime}=u\right.$ and $\left.v^{\prime}=v-1\right)$.
} 
putations, and on the other hand, the routers require additional energy for communications.

For the computations, we assume that each core involved in the execution consumes some static energy during the whole period $T$, and some dynamic energy that depends on the amount of operations, and on the speed at which these operations are executed. Let $\mathcal{A}$ be the set of active cores: $\mathcal{A}=\left\{\mathcal{C}_{u, v}, 1 \leq\right.$ $u \leq p, 1 \leq v \leq q \mid \exists 1 \leq i \leq n$, alloc $(i)=(u, v)\}$. For each core $\mathcal{C}_{u, v} \in \mathcal{A}$, let $w_{u, v}$ be its assigned work and $s_{u, v}$ its speed. The total energy consumed for computations is

$$
E^{(\mathrm{comp})}=|\mathcal{A}| \times P_{\text {leak }}^{(\mathrm{comp})} \times T+\sum_{\mathcal{C}_{u, v} \in \mathcal{A}} \frac{w_{u, v}}{s_{u, v}} \times P_{s_{u, v}}^{(\mathrm{comp})},
$$

where $T$ is the prescribed period, $P_{\text {leak }}^{(\text {comp })}$ is the leakage power dissipated together with computations, and $P_{s_{u, v}}^{(\text {comp })}$ is the dynamic power associated with speed $s_{u, v}$.

For the communications, there is also a static part due to leakage, which is paid for all cores: even if a core is not enrolled in the computation, its routers and communication links may be used to route data between remote processors. The dynamic part is directly proportional to the number of bits that are sent across each link. Hence,

$$
E^{(\mathrm{comm})}=P_{\text {leak }}^{(\mathrm{comm})} \times T+\left(\sum_{u, v} \sum_{u^{\prime}, v^{\prime}} b_{(u, v) \rightarrow\left(u^{\prime}, v^{\prime}\right)}\right) \times E^{(\mathrm{bit})},
$$

where $T$ is the period, $P_{\text {leak }}^{(\text {com })}$ is the aggregated leakage power dissipated by all routers and links, and $E^{(\text {bit })}$ is the energy to transfer a bit across neighboring cores. Finally, the total energy consumption is $E=E^{(\mathrm{comp})}+E^{(\mathrm{comm})}$.

We are ready to formally define the optimization problem:

Definition 1 (MinEnergy(T)) Given a bounded-elevation SPG and a period threshold $T$, find a mapping whose maximal cycle-time does not exceed $T$ and whose energy is minimum.

\section{Complexity results}

In this section, we assess the complexity of the MinEnergy $(T)$ problem for various instances. We classify results depending upon the target CMP, which may be uni-directional uni-line (see Section 4.1), bi-directional uni-line (see Section 4.2), or bi-directional $2 \mathrm{D}$ mesh (see Section 4.3). The only polynomial instance of MinEnergy $(T)$ is for the uni-directional uni-line CMP. In this case, we exhibit a dynamic programming algorithm that finds the optimal solution. It is worth noting that this polynomial instance becomes NP-complete for SPGs of unbounded elevation. All other problem instances are NP-hard. 


\subsection{Uni-directional uni-line CMP}

In this section, we assume that the CMP is configured as a uni-directional linear array of $q$ processors. First we provide a polynomial algorithm to solve the case of bounded-elevation SPGs. As a digression from the main focus of this paper (bounded-elevation SPGs), we prove that the problem becomes NP-hard for SPGs of unbounded elevation.

Theorem 1 The MinEnERgy $(T)$ problem on a uni-directional uni-line CMP has polynomial complexity.

Proof. We exhibit a dynamic programming algorithm which computes the optimal solution. Let $\mathcal{G}$ be a bounded-elevation SPG. First we define admissible subgraphs of $\mathcal{G}$ recursively:

- $\mathcal{G}$ is admissible;

- if a subgraph $G$ of $\mathcal{G}$ is admissible, then any subgraph of $G$ obtained by deleting one node which has no successor in $G$ is admissible too.

Let $H$ be a set of one or several nodes deleted from $G$ with this process, and let $G^{\prime}=G \backslash H$. Note that the partition $\left\{G^{\prime}, H\right\}$ is acyclic, and that any possible acyclic partition of $G$ into two subgraphs can be obtained with this construction. If we iterate the construction on $G^{\prime}$, we can build any DAG-partition of $\mathcal{G}$.

How many admissible subgraphs can we have? Let $y_{\max }$ be the maximal elevation of $\mathcal{G}$. Consider any admissible subgraph $G$. By definition, two nodes with the same $y$ coordinate are linked by a dependence. Therefore, for each value of $y$ between 1 and $y_{\max }$, there can be at most one node of elevation $y$ and without successor in $G$. Hence there are at most $n^{y_{\max }}$ admissible subgraphs (and this bound is asymptotically met for a fork-join shaped graph composed of $y_{\max }$ chains of length $n / y_{\max }$ assembled with a source and sink node).

For any admissible subgraph $G$ of $\mathcal{G}$, let $\mathcal{E}(G, k)$ be the minimum energy consumption required to execute the subgraph $G$ onto the first $k$ processors exactly. The goal is to determine $\min _{k=1}^{q} \mathcal{E}(\mathcal{G}, k)$.

The dynamic programming formulation can be expressed as:

$$
\mathcal{E}(G, k)=\min _{G^{\prime} \subseteq G}\left(\mathcal{E}\left(G^{\prime}, k-1\right) \oplus \mathcal{E}^{\mathrm{cal}}\left(G \backslash G^{\prime}\right)\right),
$$

with the initialization $\mathcal{E}(G, 1)=\mathcal{E}^{\mathrm{cal}}(G)$.

The minimum is taken over all admissible subgraphs $G^{\prime}$ such that communications between $G^{\prime}$ and $G \backslash G^{\prime}$ does not exceed the bandwidth: $\frac{C^{\text {out }}\left(G^{\prime}\right)}{B W} \leq T$, where $C^{\text {out }}\left(G^{\prime}\right)$ denotes the aggregated output data volume of $G^{\prime}$, i.e., the sum of the output data $\delta_{i}$ of all stages $S_{i} \in G^{\prime}$ which have no successor in $G^{\prime}$.

$\mathcal{E}^{\mathrm{cal}}(H)$ represents the energy consumed for the computations of the nodes in $H$ when mapped to the same processor. Given such a node set $H$, we select the minimum speed that allows for computing all the stages in $H$ within the period $T$, and we compute the corresponding energy consumption. If no such speed exists, we let $\mathcal{E}^{\mathrm{cal}}(H)=+\infty$. Finally, the $\oplus$ operator means that the energy consumed by these communications is added to the sum.

At each step, there are no more than $n^{y_{\max }}$ admissible graphs $G^{\prime}$, and therefore we have at most $n^{y_{\max }}$ values of $\mathcal{E}^{\text {cal }}(H)$ to compute, which is done in $O(n)$. Altogether, we have designed an algorithm whose worst-case complexity is $O\left(q \times n \times n^{y_{\max }}\right)$, which is polynomial since $y_{\max }$ is a constant. 


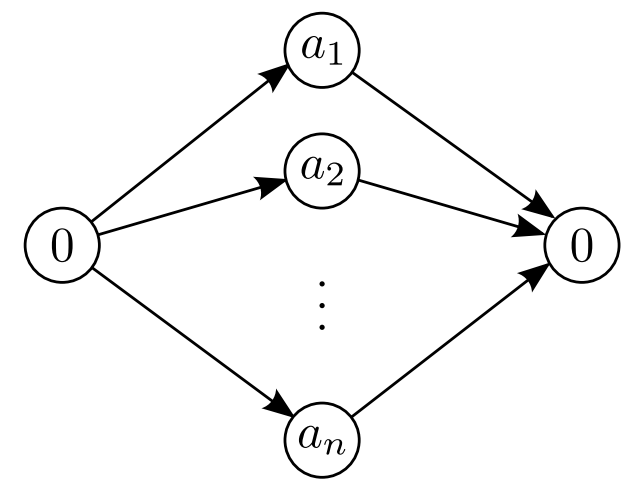

Figure 2: Unbounded-elevation SPG for the uni-directional uni-line CMP proof.

The previous theorem only holds for bounded-elevation SPGs. With unboundedelevation SPGs, the problem becomes NP-hard:

Proposition 1 The extension of MinEnergy $(T)$ to unbounded-elevation SPGs on a uni-directional uni-line CMP is NP-complete.

Proof. In fact, without any energy consideration, the simpler mono-criterion problem of matching a prescribed period is NP-complete. The associated decision problem is as follows: given a period $T$, is there a DAG-partition mapping whose period is no more than $T$ ? The problem is obviously in NP: given a period and a mapping, it is easy to check in polynomial time that it is valid by computing its period.

To establish the completeness, we use a reduction from 2-PARTITION [17]. We consider an instance $\mathcal{I}_{1}$ of 2 -PARTITION: given $n$ strictly positive integers $a_{1}, a_{2}, \ldots, a_{n}$, does there exist a subset $I$ of $\{1, \ldots, n\}$ such that $\sum_{i \in I} a_{i}=$ $\sum_{i \notin I} a_{i}$ ? Let $S=\sum_{i=1}^{n} a_{i}$.

We build an instance $\mathcal{I}_{2}$ of our problem: the application consists of a forkjoin graph of elevation $n$, as illustrated in Figure 2. We denote by $S_{0}$ the source node, $S_{n+1}$ the sink node, and $S_{i}$, for $1 \leq i \leq n$, is the $i^{t h}$ node of the fork-join. For computation costs, we have $w_{0}=w_{n+1}=0$, and $w_{i}=a_{i}$, and there are no communication costs. The platform consists of two cores which can operate only at a unique speed $s=1$. Finally, we ask whether we can achieve a period $\frac{S}{2}$.

Clearly, the size of $\mathcal{I}_{2}$ is polynomial in the size of $\mathcal{I}_{1}$. The equivalence between both problems is straightforward: if $\mathcal{I}_{1}$ has a solution $I$, then we assign $S_{0}$ and $\left\{S_{i}\right\}_{i \in I}$ on the first core, $S_{n+1}$ and $\left\{S_{i}\right\}_{i \notin I}$ on the second core. The mapping is a DAG-partition, and its period is $\frac{S}{2}$, therefore we find a solution to $\mathcal{I}_{2}$. On the other hand, if $\mathcal{I}_{2}$ has a solution, the period on each core must be exactly $\frac{S}{2}$ because the total computation requirement is $S$, and therefore we have a 2 -partition of the stages $S_{i}$, for $1 \leq i \leq n$. This concludes the proof.

\subsection{Bi-directional uni-line CMP}

Theorem 2 The MinEnERgy $(T)$ problem on a bi-directional uni-line CMP is NP-complete.

Proof. As for Proposition 1, the simpler mono-criterion problem of matching a prescribed period $T$, without any energy consideration, is NP-complete. How- 


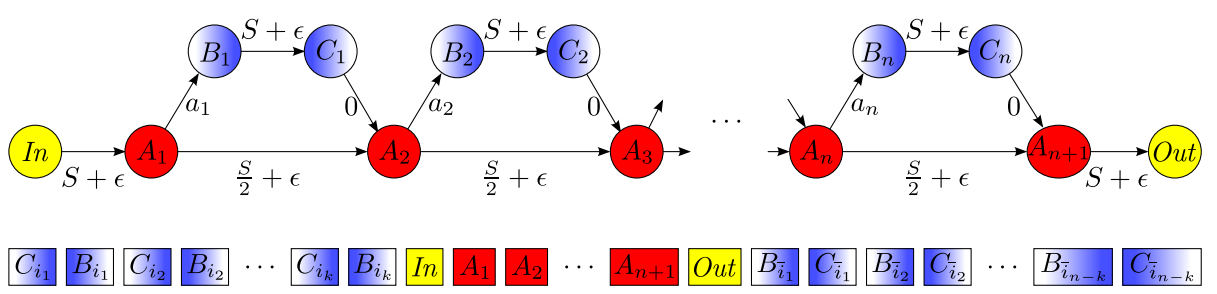

Figure 3: Bounded-elevation SPG and mapping for the bi-directional uni-line CMP proof.

ever, the reduction proof becomes quite involved, since we consider a boundedelevation SPG.

The problem is obviously in NP: given a period and a mapping, it is easy to check in polynomial time that it is valid by computing its period. To establish the completeness, we use a reduction from 2-PARTITION [17]. We consider an instance $\mathcal{I}_{1}$ of 2-PARTITION: given $n$ strictly positive integers $a_{1}, a_{2}, \ldots, a_{n}$, does there exist a subset $I$ of $\{1, \ldots, n\}$ such that $\sum_{i \in I} a_{i}=\sum_{i \notin I} a_{i}$ ? Let $S=\sum_{i=1}^{n} a_{i}$.

We build an instance $\mathcal{I}_{2}$ of our problem: the bounded-elevation SPG of the application is represented in Figure 3 . There are $3 n+3$ stages, computation costs of each stages are equal to 1 , and communication costs are depicted in the figure. The platform is a bi-directional uni-line CMP of $1 \times q$ cores, where $q=3 n+3$. Each core can operate only at a unique speed $s=1$, and the bandwidth of each link is $B W=3 S / 2+\epsilon$. Finally, we ask whether we can achieve a period of 1 . Clearly, the size of $\mathcal{I}_{2}$ is polynomial in the size of $\mathcal{I}_{1}$. We now show that instance $\mathcal{I}_{1}$ has a solution if and only if instance $\mathcal{I}_{2}$ does. First note that any solution of $\mathcal{I}_{2}$ is a one-to-one mapping, because of the constraint on the period and the computation costs of stages. Indeed, if two or more stages were mapped onto the same core, the period would be at least 2 .

Assume first that $\mathcal{I}_{1}$ has a solution, $I$. We assume that $I=\left\{i_{1}, \ldots, i_{k}\right\}$ and $\bar{I}=\{1, \ldots, n\} \backslash I=\left\{\bar{i}_{1}, \ldots, \bar{i}_{n-k}\right\}$, with $\sum_{j=1}^{k} a_{i_{j}}=\sum_{j=1}^{n-k} a_{\bar{i}_{j}}=S / 2$. For $\mathcal{I}_{2}$, we map the application graph onto the CMP as illustrated in Figure 3: for all $j \in\{1, \ldots, k\}, C_{j}$ is mapped onto $\mathcal{C}_{2 j-1}$ and $B_{j}$ onto $\mathcal{C}_{2 j}$. Then the stage In is mapped onto $\mathcal{C}_{2 k+1}$, for all $j \in\{1, \ldots, n+1\}, A_{j}$ is mapped onto $\mathcal{C}_{2 k+1+j}$, and the stage $O u t$ is mapped onto $\mathcal{C}_{2 k+n+3}$. Finally for all $j \in\{1, \ldots, n-k\}, B_{j}$ is mapped onto $\mathcal{C}_{2 k+n+2+2 j}$, and $C_{j}$ onto $\mathcal{C}_{2 k+n+2 j+3}$. The mapping is one-to-one so that the period is respected for computations. We now show that, on each link, the sum of communications does not exceed $B W$, and hence the bound on the period is not violated.

Let us first consider the link $\ell_{2 k+1+j}^{(h)}$, with $j \in\{1, \ldots, n\}$ : the amount of communications on this link is equal to $S / 2+\epsilon$ (communication from $A_{j}$ to $A_{j+1}$ ), plus at most $\sum_{i=1}^{n} a_{i}=S$ (communications from $A_{i}$ to $B_{i}$ ), therefore a total of no more than $3 S / 2+\epsilon=B W$.

Then we consider the $\operatorname{link} \ell_{2 j-1}^{(h)}$, with $j \in\{1, \ldots, k\}$ : the amount of communications is then $S+\epsilon$ (from $B_{i_{j}}$ to $C_{i_{j}}$ ) plus at most $\sum_{i \in I} a_{i}=S / 2$ (communications from $A_{i}$ to $B_{i}$, for $i \in I$ ), which is no more than $B W$. Finally, on the link $\ell_{2 j}^{(h)}$, with $j \in\{1, \ldots, k\}$, there are at most $\sum_{i \in I} a_{i}=S / 2$ communications 
(from $A_{i}$ to $B_{i}$, for $i \in I$ ). Similarly, we can prove that the bandwidth is not exceeded on link $\ell_{2 k+n+2+j}^{(h)}$, for $j \in\{1, \ldots, 2(n-k)\}$.

Only two links remain now: $\ell_{2 k+1}^{(h)}$ and $\ell_{2 k+n+2}^{(h)}$. The only communications not equals to 0 that go through $\ell_{2 k+1}^{(h)}$ are the communications from $A_{i}$ to $B_{i}$, for $i \in I$, thus the link bandwidth constraint is fulfilled. This holds true for $\ell_{2 k+n+2}^{(h)}$, reasoning with $\bar{I}$ instead of $I$. We conclude that $\mathcal{I}_{2}$ has a solution.

Assume now that $\mathcal{I}_{2}$ has a solution. We prove that the mapping is necessary similar to that of Figure 3, and that stages $B_{i}$ and $C_{i}$ must be 2-partitioned.

Let $\sigma$ be the permutation of $\{1, \ldots, n+1\}$ such that, for each $i \in\{1, \ldots, n\}$, the core assigned to $A_{\sigma(i)}$ is to the left of the one assigned to $A_{\sigma(i+1)}$. First, let us assume that there exists $i^{(0)} \in\{1, \ldots, n\}$ such that stage $I n$ is mapped between $A_{\sigma\left(i^{(0)}\right)}$ and $A_{\sigma\left(i^{(0)}+1\right)}$. Since there is a path (with edges of weight $S / 2+\epsilon$ ) going through all the $A_{\sigma(i)}$, a communication of size $S / 2+\epsilon$ occurs on all links between the core assigned to $A_{\sigma\left(i^{(0)}\right)}$ and the core assigned to $A_{\sigma\left(i^{(0)}+1\right)}$. Because of the mapping of $I n$, we deduce that there is a link on which the amount of communications is at least $3 S / 2+2 \epsilon$, which leads to a contradiction. Therefore, we showed that the core that is assigned to $I n$ is either to the left of the core assigned to $A_{\sigma(1)}$ or to the right of the core assigned to $A_{\sigma(n+1)}$. The same result holds for Out (similar proof).

Moreover note that In and Out cannot be on the same side, otherwise either the link after the core assigned to $A_{\sigma(n+1)}$ or the link before the core assigned to $A_{\sigma(1)}$ would transmit at least two communications of size $S+\epsilon$. We can assume, without loss of generality that $I n$ is mapped on the left, and Out on the right.

Similarly, for all $i \in\{1, \ldots, n\}, B_{i}$ and $C_{i}$ cannot be mapped onto a core between the core assigned to a $A_{\sigma\left(i^{\prime}\right)}$ and the one assigned to $A_{\sigma\left(i^{\prime}+1\right)}$, or In and $A_{\sigma(1)}$, or $A_{\sigma(n+1)}$ and $O u t$. The $B_{i}$ are thus mapped either to the left of In, or to the right of Out, similarly to Figure 3.

Finally, let $I$ be a subset of $\{1, \ldots, n\}$ such that $i \in I$ if and only if $B_{i}$ is mapped to the left of $I n$. Then, since the bandwith bound is not exceeded between the core assigned to $I n$ and the one assigned to $A_{\sigma(1)}$ on one hand, and between $A_{\sigma(1)}$ and $O u t$ on the other hand, we have necessarily $\sum_{i \in I} a_{i}+S+\epsilon \leq$ $3 S / 2+\epsilon$ and $\sum_{i \notin I} a_{i}+S+\epsilon \leq 3 S / 2+\epsilon$. Therefore, $\sum_{i \in I} a_{i}=\sum_{i \notin I} a_{i}=S / 2$, $\mathcal{I}_{1}$ has a solution, which concludes the proof.

\subsection{Square CMP}

In this section, we focus on square CMPs. We know from Theorem 2 that the problem is NP-hard for a $1 \times q \mathrm{CMP}$, hence for CMPs of arbitrary shapes. However, the problem complexity for a square CMP of size $p \times p$ is not a consequence of Theorem 2. We now establish this complexity:

Theorem 3 The MinEnERgy $(T)$ problem on a square CMP is NP-complete.

Proof. As for Theorem 2, the simpler mono-criterion problem of matching a prescribed period $T$, without any energy consideration, is NP-complete. The problem is obviously in NP: given a period and a mapping, it is easy to check in polynomial time that it is valid by computing its period. 
To establish the completeness, we use a reduction from 2-PARTITION [17]. We consider an instance $\mathcal{I}_{1}$ of 2 -PARTITION: given $3 n+1$ strictly positive integers $a_{1}, a_{2}, \ldots, a_{3 n+1}$, does there exist a subset $I$ of $\{1, \ldots, 3 n+1\}$ such that $\sum_{i \in I} a_{i}=\sum_{i \notin I} a_{i}$ ? Let $S=\sum_{i=1}^{3 n+1} a_{i}$.

We build the following instance $\mathcal{I}_{2}$ for our problem, re-using the construction proposed in Theorem 2. The CMP is composed of $p \times p$ cores with a single speed 1 , linked with a bandwidth $B W=3 S / 2+\epsilon$, where $p=6 n+4$. The series-parallel graph is described in Figures 4,5 and 6. All computation costs are equal to 1 . The size of blue and green communications is equal to the bandwidth, there is no communication between two $H_{i}$ widgets, neither between $E_{2}$ and $H_{6 n}$. The size of communications from $E_{1}$ to $E_{12}$ on the one side, and from $E_{13}$ to $E_{2}$, on the other side, is equal to $\epsilon$. The subgraph between $E_{12}$ and $E_{13}$ is the graph of Figure 3, replacing $n$ by $3 n+1$. Finally, we ask whether we can achieve a period of 1 . Clearly, the size of $\mathcal{I}_{2}$ is polynomial in the size of $\mathcal{I}_{1}$.

We now show that instance $\mathcal{I}_{1}$ has a solution if and only if instance $\mathcal{I}_{2}$ does. First note that any solution of $\mathcal{I}_{2}$ is a one-to-one mapping, because of the constraint on the period and the computation costs of stages. Indeed, if two or more stages were mapped onto the same core, the period would be at least 2 .

Assume first that $\mathcal{I}_{2}$ has a solution. We show that the red nodes are necessary mapped onto a linear chain of cores, and communications never escape out of this linear chain.

In each widget $G_{i, j}$, the two communications between $D_{i, 2 k-1}$ and $D_{i, 2 k}$, for $k \in\{1, \ldots, j\}$, must occur on at least 4 links and no other communications not equal to 0 can use those links, because one communication fill entirely a link, and the mapping is one-to-one. In the same way, in every widget $H_{i}$, the three communications between $P_{i, 2 k-1}, P_{i, 2 k}$ and $P_{i, 2 k+1}$, for $k \in\{1, \ldots, i\}$, must occur on at least 4 links and no other communications not equal to 0 can use those links. Moreover, there are 19 more communications of size $B W$ which thus require at least 19 more communication links. Altogether, we need at least:

$$
\begin{aligned}
\sum_{i=1}^{6 n} 4 i+3 \times(15 n-1)+(15 n+4)+19 & =2(6 n)(6 n+1)+60 n+20 \\
& =72 n^{2}+72 n+20 \\
& =2 p(p-1)-2(p-2)
\end{aligned}
$$

communication links to map all communications which are not red. If we use more links to map blue or green communications, there would be at most $2(p-$ $2)-1$ free remaining links. Now the graph contains $2(p-2)+1$ red nodes, thus a red node would be isolated, which is not possible, because not any red node has only communications of size 0 . Thus we have exactly $2(p-2)$ communication links for the red communications and $2 p \times(p-1)-2(p-2)$ communication links for blue and green communications.

Blue nodes of degree 3 are on the border of the CMP: those nodes cannot be mapped onto a corner core, because they need at least 3 free communication links, and they cannot be mapped onto a middle core either. In this case, three of four communication links would be indeed used, and the remaining empty communication link could not be used by another communication: an incoming communication could not exit through another link. The nodes $P_{3,1}, \ldots, P_{3, p-2}$ 


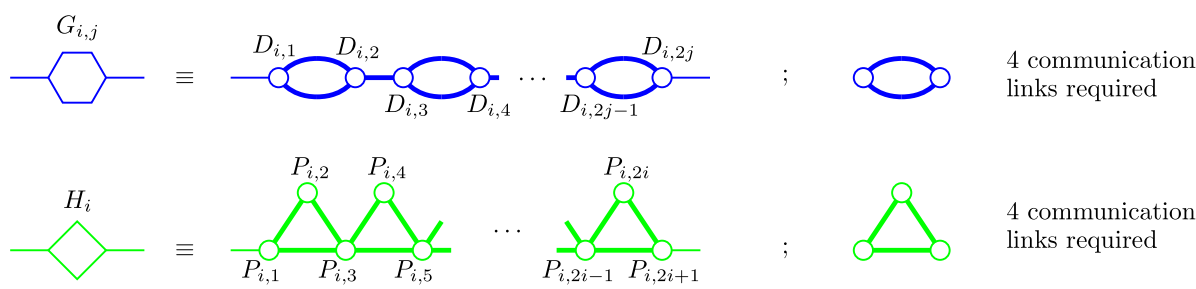

Figure 4: Widgets.

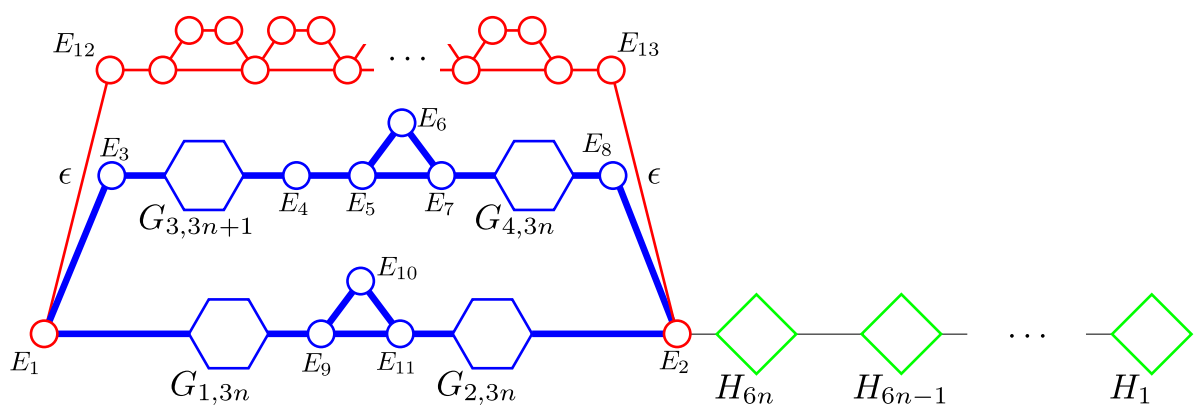

Figure 5: DAG.

of the widget $G_{3,3 n+1}$ must be mapped in order on a border, otherwise we lose at least one communication link. Without loss of generality, we can assume that they are mapped respectively onto cores $\mathcal{C}_{2,1}, \ldots, \mathcal{C}_{p-1,1}$. The communication between $P_{3,1}$ and $P_{3,2}$ must occur on links $\ell_{2,1}^{(h)}, \ell_{2,2}^{(v)}$ and $\ell_{3,1}^{(h)}$ in order not to lose any communication link. In the same way, the communication between $P_{3, p-3}$ and $P_{3, p-2}$ takes the links $\ell_{p-2,1}^{(h)}, \ell_{p-2,2}^{(v)}$ and $\ell_{p-1,1}^{(h)}$. As a result, again from the fact that we cannot lose any communication link, $E_{4}$ is mapped onto $\mathcal{C}_{p, 1}, E_{3}$ onto $\mathcal{C}_{1,1}$ then $E_{1}$ onto $\mathcal{C}_{1,2}$. In the same manner again, nodes $E_{5}, E_{7}, P_{4,1}, \ldots, P_{4, p-2}$ are mapped respectively onto cores $\mathcal{C}_{p, 2}, \ldots, \mathcal{C}_{p, p-1}, E_{8}$ onto $\mathcal{C}_{p, p}$ and $E_{2}$ onto $\mathcal{C}_{p-1, p}$.

If the graph composed of the cores on which a red node is mapped, linked with the communication links where a red communication occurs, is not a chain, $E_{1}$ and $E_{2}$ cannot be connected, because there are only $2(p-2)$ remaining communication links and the Manhattan distance between $E_{1}$ and $E_{2}$ is $2(p-2)$. Since the 2 additional nodes $E_{1}$ and $E_{2}$, and the two communications of weight $\epsilon$ do not change anything, we are in the case of proof of Theorem 2. Therefore $\mathcal{I}_{1}$ has a solution.

We assume now that $\mathcal{I}_{1}$ has a solution. We give the mapping for $n=1$ in Figure 7, which can convince us that such a mapping, where red nodes and communications are mapped onto a linear chain, can be found for any $n>1$. Then we use again the proof of Theorem 2 to conclude that $\mathcal{I}_{2}$ has a solution, and hence conclude the proof. 


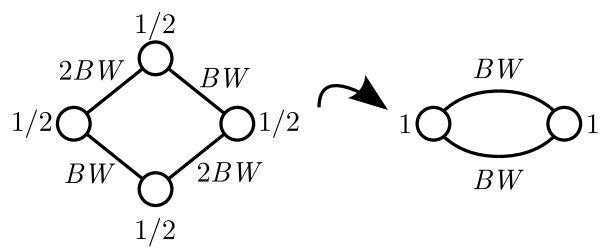

Figure 6: SPG to DAG.
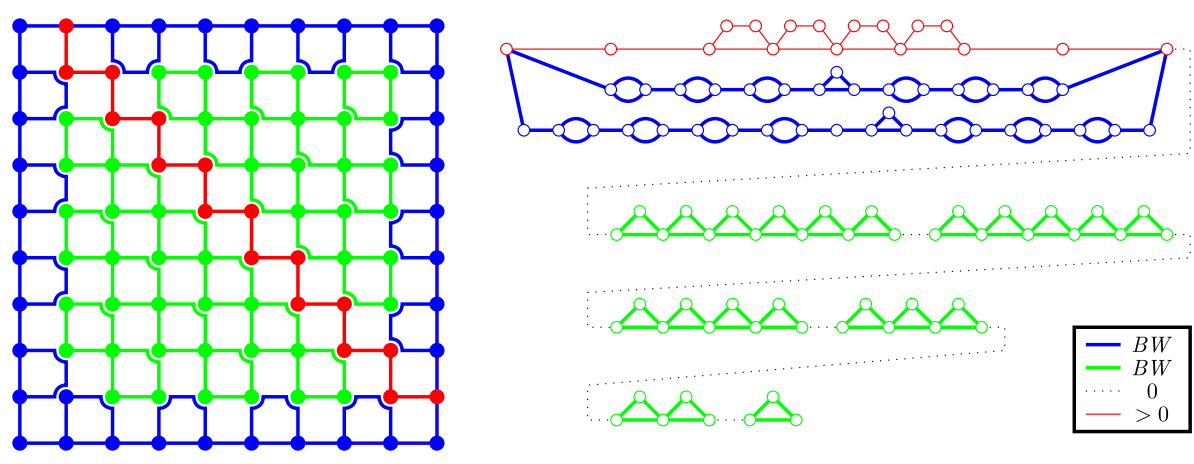

Figure 7: Example $10 \times 10$.

\subsection{Integer linear program}

The general problem of finding the optimal DAG-partition mapping, for a given period, has been shown to be NP-hard. However, we formulate in this section the problem as an integer linear program (ILP), which allows us to find the optimal solution of the problem (in exponential time) for small problem instances. Actually, this ILP can also find the optimal general mapping (without the restriction of DAG-partition mappings), by removing the DAG-partition constraint from the program.

Unfortunately, because of the large number of variables needed to express communication paths in the CMP, we were unable to obtain results on a platform larger than a $2 \times 2 \mathrm{CMP}$ with ILOG CPLEX [15].

\subsection{Constants}

We first define the set of constant values that define our problem. The application is composed of $n$ stages $S_{1}, \ldots, S_{n}$, and a set of edges $\mathcal{E}$ :

- for $1 \leq i \leq n, w(i)$ is the amount of computations of node $S_{i}$, i.e., it corresponds to the $w_{i}$ parameter;

- for $1 \leq i, j \leq n, \ell(i, j)=1$ if there is a link between $S_{i}$ and $S_{j}$ (i.e., if $\left.L_{i, j} \in \mathcal{E}\right)$, and then $\delta(i, j)$ is the amount of communications between the two stages (it corresponds to the $\delta_{i, j}$ parameter); otherwise $\ell(i, j)=$ $\delta(i, j)=0$;

- we define $\ell^{*}$ as the transitive closure of $\ell$, i.e., for $1 \leq i, j \leq n, \ell^{*}(i, j)=1$ if there is a dependence path from $S_{i}$ to $S_{j}$, otherwise $\ell^{*}(i, j)=0$. 
For the platform, we consider a $p \times q \mathrm{CMP}$, and we need to compute beforehand the energy consumed by a core when running at a supply voltage $U_{k}$, for $1 \leq k \leq x$. Note that the corresponding speed of the core is always the highest speed for the voltage.

- for $1 \leq k \leq x, s(k)$ is the speed of a core operated at voltage $U_{k}$;

- for $1 \leq k \leq x, \operatorname{Edyn}(k)=U_{k}^{2}$ (square of the voltage), and $\operatorname{Estat}(k)=$ $I^{\text {(leak) }} \times U_{k} \times T$ (i.e., the static energy consumption of a core operated at voltage $U_{k}$ ).

Finally, $B W$ is the link bandwidth, and $T$ is the bound on the period.

\subsection{Variables}

Now that we have defined the constants that define our problem, we define unknown variables to be computed:

- for $1 \leq i \leq n, 1 \leq k \leq x, 1 \leq u \leq p$ and $1 \leq v \leq q, x_{i, k, u, v}$ is a boolean variable equal to 1 if stage $S_{i}$ is mapped onto core $\mathcal{C}_{u, v}$, operated at voltage $U_{k}$, and 0 otherwise; there are $n \times x \times p \times q$ such variables;

- for $1 \leq k \leq x, m_{k, u, v}$ is a boolean variable equal to 1 if core $\mathcal{C}_{u, v}$ is operated at voltage $U_{k}$, and 0 otherwise; there are $x \times p \times q$ such variables;

- for $1 \leq i, j \leq n, 1 \leq u \leq p$ and $1 \leq v \leq q, c_{i, j, u, v}^{N}$ (resp. $c_{i, j, u, v}^{S}, c_{i, j, u, v}^{W}$ and $c_{i, j, u, v}^{E}$ ) is a boolean variable equal to 1 if there is a communication for link $L_{i, j}$ between core $\mathcal{C}_{u, v}$ and its north (resp. south, west, east) neighbor $\mathcal{C}_{u-1, v}$ (resp. $\mathcal{C}_{u+1, v}, \mathcal{C}_{u, v-1}, \mathcal{C}_{u, v+1}$ ) and 0 otherwise; for $u=1$ (resp. $u=p, v=1, v=q$ ), we enforce that the variable is set to 0 (no possible communication because of the borders of the CMP).; there are $4 \times n^{2} \times p \times q$ such variables.

For convenience, we note $c_{i, j, u, v}^{*}=c_{i, j, u, v}^{N}+c_{i, j, u, v}^{S}+c_{i, j, u, v}^{W}+c_{i, j, u, v}^{E}$.

\subsection{Constraints}

Finally, we must write all constraints involving our constants and variables. In the following, unless stated otherwise, $i, j, i^{\prime}$ span $[1, n]$ (stage indices); $u, u^{\prime}$ span $[1, p]$ and $v, v^{\prime} \operatorname{span}[1, q]$ (processor indices), and finally $k, k^{\prime} \operatorname{span}[1, x]$ (mode indices). First we need constraints to guarantee that the allocation of stages to cores is a valid allocation, and that the voltage of each core is correctly set.

- $\forall i, k, \sum_{u, v} x_{i, k, u, v}=1$ : each stage is allocated to exactly one core;

- $\forall k, u, v, m_{k, u, v} \geq \sum_{i} x_{i, k, u, v}$ : if stage $S_{i}$ is mapped onto $\mathcal{C}_{u, v}$ operated at a voltage $U_{k}$, then $\mathcal{C}_{u, v}$ must be operated at a voltage $U_{k}$;

- $\forall u, v \sum_{k} m_{k, u, v} \leq 1$ : each core is operated at no more than one voltage (either the core is on and the sum equals 1 , or it is off and the sum equals 0$)$.

Then, we need to ensure that communications are correctly scheduled, by enforcing constraints on the $c_{i, j, u, v}$ variables. 
- $\forall i, j, u, v, c_{i, j, 1, v}^{N}=0, c_{i, j, p, v}^{S}=0, c_{i, j, u, 1}^{W}=0$, and $c_{i, j, u, q}^{E}=0$ : no communication is allowed outside the borders of the CMP;

- $\forall i, j, u, v, c_{i, j, u, v}^{*} \leq \ell(i, j)$ : there is no communication from $S_{i}$ to $S_{j}$ if there is no dependence constraint between these two stages;

- $\forall i, j, k, u, v, x_{i, k, u, v}+x_{j, k, u, v}+c_{i, j, u, v}^{*} \leq 2$ : this condition enforces that if $S_{i}$ and $S_{j}$ are mapped onto the same core, $\mathcal{C}_{u, v}$, then there is no communication for link $L_{i, j}$ initiated from $\mathcal{C}_{u, v}$;

- $\forall i, j, k, c_{i, j, u, v}^{*} \geq x_{i, k, u, v}+\sum_{k^{\prime},(u, v) \neq\left(u^{\prime}, v^{\prime}\right)} x_{j, k^{\prime}, u^{\prime}, v^{\prime}}+\ell(i, j)-2$ : this initiates the communication for $L_{i, j}$ if $S_{i}$ and $S_{j}$ are mapped onto two distinct cores; the communication must occur into one of the directions $(\mathrm{N}, \mathrm{S}, \mathrm{W}$ or $\mathrm{E})$;

- $\forall i, j, u<p, v, c_{i, j, u, v}^{S} \leq c_{i, j, u+1, v}^{*}+\sum_{k} x_{j, k, u+1, v} \leq 2-c_{i, j, u, v}^{S}$ : if there was a communication initiated from $\mathcal{C}_{u, v}$ to the south for $L_{i, j}\left(c_{i, j, u, v}^{S}=1\right)$, then either we reach the destination core $\left(\sum_{k} x_{j, k, u+1, v}=1\right)$, or the communication must be forwarded on one of the links from $\mathcal{C}_{u+1, v}\left(c_{i, j, u+1, v}^{*}=\right.$ 1 ); otherwise there is no constraint; these constraints express both the forwarding of communications and the stopping condition;

- there are similar constraints for other directions:

$\forall i, j, u>1, v, c_{i, j, u, v}^{N} \leq c_{i, j, u-1, v}^{*}+\sum_{k} x_{j, k, u-1, v} \leq 2-c_{i, j, u, v}^{N} ;$

$\forall i, j, u, v<q, c_{i, j, u, v}^{E} \leq c_{i, j, u, v+1}^{*}+\sum_{k} x_{j, k, u, v+1} \leq 2-c_{i, j, u, v}^{E} ;$

$\forall i, j, u, v>1, c_{i, j, u, v}^{W} \leq c_{i, j, u, v-1}^{*}+\sum_{k} x_{j, k, u, v-1} \leq 2-c_{i, j, u, v}^{W}$.

A set of constraints express the fact that no cycle can occur in the communications:

- $\forall i, j, p>u>1, q>v>1, c_{i, j, u+1, v}^{N}+c_{i, j, u-1, v}^{S}+c_{i, j, u, v-1}^{E}+c_{i, j, u, v+1}^{W} \leq \sum_{k} x_{i, k, u, v}$;

- $\forall i, j, q>v>1, c_{i, j, 2, v}^{N}+c_{i, j, 1, v-1}^{E}+c_{i, j, 1, v+1}^{W} \leq \sum_{k} x_{i, k, 1, v}$;

- $\forall i, j, q>v>1, c_{i, j, p-1, v}^{S}+c_{i, j, p, v-1}^{E}+c_{i, j, p, v+1}^{W} \leq \sum_{k} x_{i, k, p, v}$;

- $\forall i, j, p>u>1, c_{i, j, u+1,1}^{N}+c_{i, j, u-1,1}^{S}+c_{i, j, u, 2}^{W} \leq \sum_{k} x_{i, k, u, 1}$;

- $\forall i, j, p>u>1, c_{i, j, u+1, q}^{N}+c_{i, j, u-1, q}^{S}+c_{i, j, u, q-1}^{E} \leq \sum_{k} x_{i, k, u, q}$;

- $\forall i, j, c_{i, j, 2,1}^{N}+c_{i, j, 1,2}^{W} \leq \sum_{k} x_{i, k, 1,1}$;

- $\forall i, j, c_{i, j, p-1,1}^{S}+c_{i, j, p, 2}^{W} \leq \sum_{k} x_{i, k, p, 1}$;

- $\forall i, j, c_{i, j, 2, q}^{N}+c_{i, j, 1, q-1}^{E} \leq \sum_{k} x_{i, k, 1, q}$;

- $\forall i, j, c_{i, j, p-1, q}^{S}+c_{i, j, p, q-1}^{E} \leq \sum_{k} x_{i, k, p, q}$.

Another constraint expresses the fact that the mapping is a DAG-partition:

- $\forall i, i^{\prime}, j, k, u, v, x_{i^{\prime}, k, u, v} \geq \ell_{i, i^{\prime}}^{*} \times \ell_{i^{\prime}, j}^{*} \times\left(x_{i, k, u, v}+x_{j, k, u, v}-1\right)$ : if two stages $S_{i}$ and $S_{j}$ are mapped onto the same core $\mathcal{C}_{u, v}$, then any stage $S_{i^{\prime}}$ which has an incoming dependency from $S_{i}$ and an outgoing dependency from $S_{j}$ must be mapped onto the same core, otherwise there would be a cycle in the partition. 
Finally, we express the fact that the constraint on the period is fulfilled:

- $\forall u, v, k, \sum_{i} x_{i, k, u, v} \times w(i) \leq T \times m_{k, u, v} \times s(k)$ : constraint on computations;

- $\forall u, v \sum_{i, j} c_{i, j, u, v}^{N} \times \delta(i, j) \leq T \times B W$ : constraint on north communications;

- $\forall u, v \sum_{i, j} c_{i, j, u, v}^{S} \times \delta(i, j) \leq T \times B W$ : constraint on south communications;

- $\forall u, v \sum_{i, j} c_{i, j, u, v}^{W} \times \delta(i, j) \leq T \times B W$ : constraint on west communications;

- $\forall u, v \sum_{i, j} c_{i, j, u, v}^{E} \times \delta(i, j) \leq T \times B W$ : constraint on east communications.

\subsection{Objective function}

We aim at minimizing the energy consumption, which writes:

$$
\min \left(\begin{array}{c}
\sum_{u, v}\left(\sum_{k} m_{k, u, v} \times \operatorname{Estat}(k)\right. \\
\left.+\sum_{i, k} x_{i, k, u, v} \times w(i) \times \operatorname{Edyn}(k)\right) \\
+\sum_{u, v, i, j} c_{i, j, u, v}^{*} \times \delta(i, j) \times E^{(\mathrm{bit})}
\end{array}\right) .
$$

The objective function is linear, as well as all the constraints. Since the variables are boolean, this is an integer linear program.

\section{Heuristics}

In this section, we describe the four heuristics that we have designed and implemented, thus providing practical solutions to the $\operatorname{MinEnERGY}(T)$ problem. The first heuristic, Random (Section 5.1), performs a random mapping, and it is used for comparison purposes. Then we propose a greedy heuristic, Greedy, in Section 5.2, and a heuristic based on a two-dimensional dynamic programming algorithm, DPA2D, in Section 5.3. Finally, we use the optimal dynamic programing algorithm for uni-directional uni-line CMPs (see Section 4.1) to design heuristic DPA1D (Section 5.4).

\subsection{Random heuristic}

This first heuristic calls a procedure which works in two steps. The procedure first randomly builds a DAG-partition of the initial SPG, while ensuring that the period is matched for computations: we choose randomly a speed for the core which will handle the current subgraph $G$ (initially, the source of the SPG), and we keep a list of stages of the SPGs that can be added to $G$ while maintaining a DAG-partition. We pick a stage from this list randomly as long as computations do not exceed the period. When moving to the next core, we choose the first stage in the current list and iterate. In the second step, we decide randomly on which core each subgraph is mapped, and communications are done following a $X Y$ routing: a communication from $\mathcal{C}_{u, v}$ to $\mathcal{C}_{u^{\prime}, v^{\prime}}$ follows horizontal links from $\mathcal{C}_{u, v}$ to $\mathcal{C}_{u^{\prime}, v}$, and then vertical links from $\mathcal{C}_{u^{\prime}, v}$ to $\mathcal{C}_{u^{\prime}, v^{\prime}}$. If the period is not exceeded on any communication link, then the mapping is valid, otherwise there is no solution.

For each problem instance, Random calls ten times this procedure, and keeps the solution which minimizes the energy consumption, if there is at least one valid solution; otherwise it fails. 


\subsection{Greedy heuristic}

Given a speed $s \in \mathrm{S}$, this heuristic greedily assigns the SPG onto the platform, on which all cores are running at speed $s$. The greedy assignment is done through procedure greedy $(s)$. The idea is to try all possible speed values, and to keep the best solution.

The greedy procedure greedy $(s)$ works as follows: we keep a list of cores which are ready to be processed, and for each core, a list of outgoing communications. Initially, the only core in the list is $\mathcal{C}_{1,1}$, and we assign to this core the source stage $S_{1}$. The corresponding list of outgoing communications corresponds to the successors of $S_{1}$ in the SPG, and they are sorted by non-increasing communication volume.

When we process a core $\mathcal{C}_{u, v}$, we successively try to add some of the successors (from the communication list) to this same core (we start with no other stage, until the communication list is empty or the period is exceeded for computations on $\mathcal{C}_{u, v}$ ).

For each set of stages mapped onto $\mathcal{C}_{u, v}$ and the corresponding list of outgoing communications, we greedily share these communications between neighboring cores $\mathcal{C}_{u, v+1}$ and $\mathcal{C}_{u+1, v}$ : communications are taken from the sorted list and assigned to the core which has currently the smallest amount of incoming communications. Then, we check that the partitioning is correct (no cycles in the dependence graph, i.e., we have a DAG-partition), and we check whether the bound on the period is achieved, both for computations and communications. If it is correct, we save the current solution before adding one more stage onto core $\mathcal{C}_{u, v}$ and iterating with one more stage on $\mathcal{C}_{u, v}$.

At the end of the iteration, we keep the last valid (saved) solution, i.e., the valid solution with the most number of stages onto $\mathcal{C}_{u, v}$. Cores $\mathcal{C}_{u, v+1}$ and $\mathcal{C}_{u+1, v}$ are then added to the list of ready cores, together with the list of outgoing communications (i.e., the stages that can either be assigned to this cores, or forwarded to the neighboring cores).

The procedure finishes when the core list is empty (i.e., there are no pending outgoing communications, which means that all stages have been processed). Otherwise, the heuristic fails, and we move to the next speed. The energy for the mapping obtained with a given speed is computed by first downgrading the speed of each core, if possible: the procedure returns the mapping, and then we compute the amount of computations on each core, and set the core to the slowest possible speed, in order to save energy. Cores which are not used are turned off. Finally, the Greedy heuristic selects the mapping which corresponds to the lowest energy consumption.

\subsection{D dynamic programming algorithm}

This heuristic, called DPA2D, starts by mapping the initial SPG onto a $x_{\max } \times$ $y_{\max }$ grid, following the labels of the nodes (see Section 3.1). Then, this grid is mapped onto the CMP, thanks to a double nested dynamic programming algorithm.

First, we perform a dynamic programming algorithm to cut the grid into a set of columns, which are to be mapped onto a column of cores. Let $\mathcal{E}(m, v, D)$ be the optimal energy consumption to compute the first $m$ levels of the SPG (i.e., all stages $S_{i}$ with $x_{i} \leq m$ ), using $v$ columns of cores, regardless of the 
outgoing communications. $D$ is then the corresponding distribution of outgoing communications, i.e., a list of triples $(y, b, i)$, where $y$ is the row from which communication is outgoing (i.e., the communication is initiated by core $\mathcal{C}_{y, v}$ ), $b$ is the amount of data, and $S_{i}$ is the destination stage. We enforce these communications to go through $\mathcal{C}_{y, v+1}$, and then the communication will be redistributed to the destination core through vertical links. The solution is $\mathcal{E}\left(x_{\max }, q, D\right)$, and the recurrence is written as:

$$
\mathcal{E}(m, v, D)=\min _{m^{\prime}<m}\left(\begin{array}{r}
\mathcal{E}\left(m^{\prime}, v-1, D^{\prime}\right)+\mathcal{E}^{\mathrm{comm}}\left(D^{\prime}\right) \\
+\mathcal{E}^{\mathrm{col}}\left(m^{\prime}+1, m, D^{\prime}, D\right)
\end{array}\right)
$$

with the initialization $\mathcal{E}(m, 1, D)=\mathcal{E}^{\mathrm{col}}(1, m, \emptyset, D)$.

$D^{\prime}$ is the distribution of outgoing communications corresponding to the $m^{\prime}$ which leads to the optimal energy consumption, i.e., obtained with $\mathcal{E}\left(m^{\prime}, v-\right.$ $\left.1, D^{\prime}\right)$.

$\mathcal{E}^{\mathrm{comm}}\left(D^{\prime}\right)$ is the energy consumption induced by communications from column $v-1$ to column $v$ (on horizontal links), given the distribution $D^{\prime}$ of outgoing communications of column $v+1$. If the bandwidth is exceeded on one of these horizontal links (i.e., $\exists 1 \leq y \leq p$ such that $\left.\sum_{(y, b, i) \in D^{\prime}} b>B W\right)$, we set $\mathcal{E}^{\mathrm{comm}}\left(D^{\prime}\right)=+\infty$.

$\mathcal{E}^{\mathrm{col}}\left(m_{1}, m_{2}, D^{\prime}, D\right)$ is the optimal energy consumption of the column of the CMP which is processing stages $S_{i}$ with $m_{1} \leq x_{i} \leq m_{2}$ : it accounts both for computations, and for vertical communications in the column, given the distribution of outgoing communications of the previous column, $D^{\prime}$. The distribution of outgoing communications of this column is then $D$. Note that in the recurrence, $D$ is an output of $\mathcal{E}^{\mathrm{col}}\left(m^{\prime}+1, m, D^{\prime}, D\right)$, while $D^{\prime}$ is an output of $\mathcal{E}\left(m^{\prime}, v-1, D^{\prime}\right)$. The values of $\mathcal{E}^{\text {col }}$ (and therefore, distribution $D$ ) are computed thanks to another dynamic programming algorithm: we compute $\mathcal{E}_{\left(m_{1}, m_{2}, D^{\prime}, D\right)}^{\text {col }}(g, u)$, which corresponds to the mapping of stages $S_{i}$, with $m_{1} \leq x_{i} \leq m_{2}$ and $y_{i} \leq g$, onto the $u$ first cores of a column of the CMP. As before, $D^{\prime}$ is an input, it corresponds to the distribution of outgoing communications arriving into the current column, while $D$ is the distribution of outgoing communications of the current column for the solution which minimizes the energy consumption. Then we have $\mathcal{E}^{\mathrm{col}}\left(m_{1}, m_{2}, D^{\prime}, D\right)=\mathcal{E}_{\left(m_{1}, m_{2}, D^{\prime}, D\right)}^{\mathrm{col}}\left(y_{\max }, p\right)$.

For the distribution within a column, the recurrence writes:

$$
\mathcal{E}_{\left(m_{1}, m_{2}, D^{\prime}, D\right)}^{\mathrm{col}}(g, u)=\min _{g^{\prime} \leq g}\left(\begin{array}{r}
\mathcal{E}_{\left(m_{1}, m_{2}, D^{\prime}, D\right)}^{\mathrm{col}}\left(g^{\prime}, u-1\right) \\
+\mathcal{E}_{\left(m_{1}, m_{2}, D\right)}^{\mathrm{cal}}\left(g^{\prime}+1, g\right) \\
+\mathcal{E}_{\left(m_{1}, m_{2}, D^{\prime}\right)}^{\mathrm{ver}}\left(g^{\prime}+1, g, u-1\right)
\end{array}\right),
$$

with the initialization $\mathcal{E}_{\left(m_{1}, m_{2}, D^{\prime}, D\right)}^{\mathrm{col}}(0, u)=0$, and no outgoing communications from row 1 to row $u$, except the communications from $D^{\prime}$ that must be forwarded to the next column.

$\mathcal{E}_{\left(m_{1}, m_{2}, D^{\prime}\right)}^{\mathrm{ver}}\left(g^{\prime}+1, g, u-1\right)$ is the energy consumption of the vertical communications between cores $u-1$ and $u$ in the column. These communications can either come from two dependent stages of the column, or be forwarded from the previous column $\left(D^{\prime}\right)$. If the bandwidth of the link is exceeded, we set the value to $+\infty$.

Finally, $\mathcal{E}_{\left(m_{1}, m_{2}, D\right)}^{\text {cal }}\left(g^{\prime}+1, g\right)$ is the optimal energy consumption of a core which is computing all stages $S_{i}$ such that $m_{1} \leq x_{i} \leq m_{2}$, and $g^{\prime}+1 \leq y_{i} \leq g$. 
If the period cannot be respected, or if the corresponding partition does not respect the DAG-partition constraint, the value is set to $+\infty$. Moreover, this function is adding to distribution $D$ the communications from a stage $S_{i}$ to another stage $S_{j}$, with $x_{j}>m_{2}$. These communications will occur on row $u$.

Note that in the recursive computation of $\mathcal{E}^{\mathrm{col}}$, we can have $g^{\prime}=g$, which means that no stage is assigned to core $\mathcal{C}_{u, v}$. This may happen if there are not enough stages in the column, or if this would save communications.

\subsection{D dynamic programming algorithm}

The last heuristic, DPA1D, builds upon the theoretical results of Section 4 . Indeed, all problem instances are NP-hard, except the mapping of a SPG onto a uni-directional uni-line CMP. This heuristic configures the CMP as a unidirectional uni-line CMP with $r=p \times q$ cores, by embedding it into the bidirectional platform as a snake:

$$
\begin{aligned}
\mathcal{C}_{1,1} & \rightarrow \mathcal{C}_{1,2} \rightarrow \cdots \rightarrow \quad \mathcal{C}_{1, q} \\
\mathcal{C}_{2,1} & \leftarrow \cdots \leftarrow \mathcal{C}_{2, q-1} \leftarrow \mathcal{C}_{2, q} \\
\downarrow & \downarrow \\
\mathcal{C}_{3,1} & \rightarrow \mathcal{C}_{3,2} \rightarrow \ldots
\end{aligned}
$$

Then, we compute the optimal solution of the dynamic programming algorithm of Theorem 1 with $r=p \times q$ cores, and the mapping is done along the snake; no other communication link is used.

Note that if the SPG is a linear chain, or if there is no communication cost, then this heuristic is optimal, since any other solution could not exploit the communication links discarded with the snake structure. However, DPA1D may take wrong decisions when communications are intensive, since it is restricted to a subset of communication links. Moreover, its complexity of $O\left(p \times q \times n \times n^{y_{\max }}\right)$ makes it intractable for SPGs with large $y_{\max }$.

\section{Experiments}

This section reports experimental results assessing the performance of the various heuristics. As for the applications, we use the StreamIt suite [45], and variants of these workflows with the same graph structures but different computation-to-communication ratios. As for the target platform, we use $4 \times 4$ and $6 \times 6$ CMP grids, whose hardware characteristics are representative of state-of-the-art devices. The source code for all experiments is publicly available at [42].

\subsection{Experimental setting}

First we detail the parameters used for the workflows, and for the CMP configuration.

Streaming applications. There are 12 workflows in the StreamIt suite [45]. Their main characteristics are summarized in Table 1, where we give the size $n$, the maximum label values $y_{\max }$ and $x_{\max }$, and their computation-to-communication ratio $(C C R)$, defined as the sum $\sum_{i=1}^{n} w_{i}$ of all computations over the sum $\sum_{L_{i, j} \in \mathcal{E}} \delta_{i, j}$ of all communications. We observe in Table 1 that all workflows 
have a large CCR, hence are compute-intensive rather than data-intensive. In the experiments, we first use the workflows as such, with the original CCR values. and then we scale communication weights (the $\delta_{i, j}$ ) to change each CCR successively to 10,1 , and 0.1 , so as to assess the impact of the communications on the performance of the heuristics.

\begin{tabular}{|c|l|c|c|c|c|}
\hline Index & Name & $n$ & $y_{\max }$ & $x_{\max }$ & CCR \\
\hline 1 & Beamformer & 57 & 12 & 12 & 537 \\
2 & ChannelVocoder & 55 & 17 & 8 & 453 \\
3 & Filterbank & 85 & 16 & 14 & 535 \\
4 & FMRadio & 43 & 12 & 12 & 330 \\
5 & Vocoder & 114 & 17 & 32 & 38 \\
6 & BitonicSort & 40 & 4 & 23 & 6 \\
7 & DCT & 8 & 1 & 8 & 68 \\
8 & DES & 53 & 3 & 45 & 7 \\
9 & FFT & 17 & 1 & 17 & 17 \\
10 & MPEG2-noparser & 23 & 5 & 18 & 9 \\
11 & Serpent & 120 & 2 & 111 & 9 \\
12 & TDE & 29 & 1 & 29 & 12 \\
\hline
\end{tabular}

Table 1: Characteristics of the StreamIt workflows.

CMP configuration. For processor speeds and power consumption, we use the model of the Intel Xscale [23], following [13, 11, 36]. There are five speeds for each core: $s_{u, v}=(0.15,0.4,0.6,0.8,1) G H z$, with power consumption $P_{s_{u, v}}^{(\text {comp })}=(80,170,400,900,1600) m W$. We assume that the power consumption of the processor when it is idle is $P_{\text {leak }}^{(\mathrm{comp})}=80 \mathrm{~mW}$. We use 16-byte wide communication links [39], whose bandwidths are $B W=16 \times 1.2$ Gbytes, which is reasonable according to [39]. Note that from the communication prospective, decreasing CCR has the same effect on the results as decreasing the width of the communication link below 16 bytes. The link energy is assumed to be between 1 and 10 picojoule per bit [9]; we fix $E^{(\text {bit })}=6 p J$. Finally, we use $P_{\text {leak }}^{(\text {comm })}=0$ without loss of generality (because for all heuristics the same quantity $P_{\text {leak }}^{(\text {comm })} \times T$ will be added to the total energy).

Period bound $T$. We need to find a meaningful value of $T$ for each workflow. Indeed, if $T$ is too large, all heuristics will map all stages onto a single processor running at the slowest speed, while if $T$ is too small, all heuristics will fail. We choose $T$ as follows: for each workflow, we start with $T=1 s$. With such a period, we observe that at least one heuristic succeeds. Then we iteratively divide the period by a factor of 10 and run all heuristics under this new value until all heuristics fail. We retain the period as the penultimate value, which is the last one before total failure. Note that this value depends upon the workflow, and that it is chosen to give some tightness to the mapping problem: at least one heuristic succeeds to find a mapping that matches the bound $T$, but none does for $T / 10$. 


\subsection{Experimental results}

In the following eight graphs, we plot the energy computed by the four heuristics for each application, given a CMP size $(4 \times 4$ or $6 \times 6)$ and a CCR ratio (set to the original value, 10,1 and 0.1 ). On the horizontal $x$ axis, each group corresponds to an application, and $x$ is the number of the application in Table 1 . On the vertical axis, we plot the energy found by each heuristic, normalized by the minimum value obtained over all heuristics (so that the best heuristic returns 1 , and the other ones return higher values). The DPA1D heuristic fails to return a solution for the first four applications, because there are too many possible splits to explore, and it is not plotted for those applications. More generally, each time a heuristic fails on a given application, it does not appear on the corresponding graph.

$\mathbf{4} \times \mathbf{4}$ CMP grid. Results for a $4 \times 4$ CMP grid are given in Figure 8 . When computations are predominant, i.e., when the CCR is set to its original value, or uniformly equal to 10, we observe that Greedy, DPA2D and DPA1D return similar results, and that Random always is within a factor of two. We also observe that DPA2D often fails on graphs with small elevation (linear graphs), because it wastes a lot of cores. For instance, if the application is exactly a pipeline (workflows numbered 7, 9 and 12), DPA2D can only enroll 4 cores over the 16 that are available. This fact holds true irrespective of the CCR.

When communications are more important, i.e., when the CCR is uniformly set to 1 or 0.1 , Random gets much worse than the other heuristics: if its does not fail, its energy is between 2 and 4 times worse than the best one. In a general manner, we see that DPA2D is the best heuristic when the application graph has a high elevation. DPA2D can be better than DPA1D even for applications with a low elevation, because it better handles communications. For example, the workflow numbered 10 requires some communications between stages with very different length, and DPA1D pays this communication several times.

We point out that DPA1D is the only successful heuristic for the workflow 11, whatever the CCR ratio is. This workflow fits very well with the main design idea of DPA1D: it is a pipeline-like graph (its elevation is only 2) with numerous stages. The other heuristics fail to find a good load-balance of computations and communications for this application.

Altogether, Greedy seems to be a general-purpose heuristic that succeeds on most graphs, and it is always superior to Random. On the contrary, DPA1D and DPA2D are "specialized" heuristics, the former is very efficient for long and almost linear graphs but not good for fat graphs of large elevation, and the latter behaving just as the opposite.

$6 \times 6 \mathbf{C M P}$ grid. Results for a $6 \times 6 \mathrm{CMP}$ grid are given in Figure 9 . Because the target grid is larger, it is easier to find a mapping that matches the period bound, especially for applications with a small number of stages. This is quantified in Table 2, where we report the number of failures for each heuristic. Otherwise, the conclusion remains more or less the same, with Greedy always successful but also always inferior to one of the two specialized heuristics, either DPA1D or DPA2D, depending upon the graph shape. 

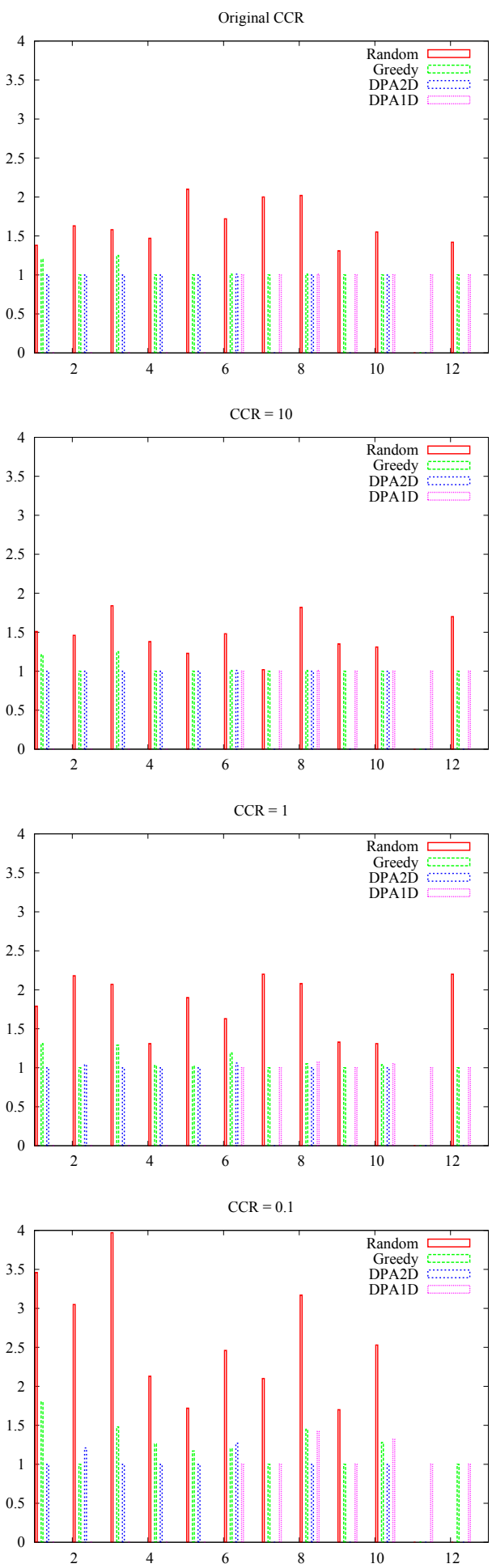

Figure 8: Normalized energy on the set of applications for a $4 \times 4$ CMP grid. 

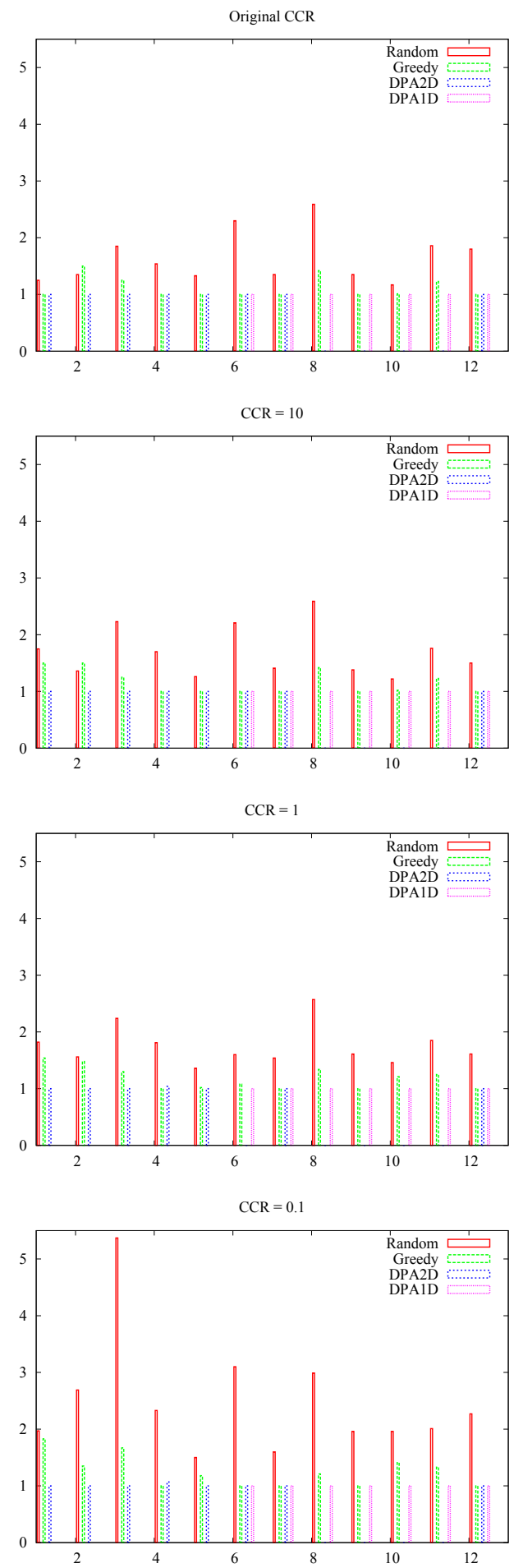

Figure 9: Normalized energy on the set of applications for a $6 \times 6 \mathrm{CMP}$ grid. 


\begin{tabular}{|c|c|c|c|c|}
\hline Platform size & Random & Greedy & DPA2D & DPA1D \\
\hline $4 \times 4$ & 5 & 4 & 16 & 20 \\
$6 \times 6$ & 0 & 0 & 17 & 20 \\
\hline
\end{tabular}

Table 2: Number of failures for each heuristic (out of 48 instances per CMP grid size).

\section{Conclusion}

This paper contributes to the efficient utilization of multicores by considering an important class of streaming applications that can be modeled by a series-parallel graph, and studying the problem of mapping these applications to 2-dimensional tiled CMP architectures. The objective of the mapping is to minimize the energy consumption while maintaining a given level of performance, reflected by the rate of processing the data streams. Both processing and communication capabilities and power consumption are considered during the mapping but it is assumed that only the processing power can be managed through dynamic voltage and frequency scaling. We will consider systems in which the communication power can also be managed in future work.

From a theoretical angle, we showed that most of the bi-criteria mapping problems were NP-complete, with the notable exception of uni-directional uniline CMPs, for which an elaborated dynamic programming algorithm returns the optimal solution. The latter result holds true only for bounded-elevation SPGs, and the problem becomes NP-complete otherwise, which provides yet another evidence of the interest to restrict to particular graph structures rather than to deal with arbitrary DAGs. We strongly believe that bounded-elevation SPGs represent a very interesting trade-off, as they combine a large practical significance while being amenable to rigorous analysis. From a practical angle, the simulations conducted with the StreamIt suite [45] confirmed the efficiency of the main design principles underlying the various heuristics. While Greedy is the most robust approach, it is always superseded by one of the two specialized algorithms, either DPA1D for long pipeline-like graphs or DPA2D for fat graphs of large elevation.

Our ongoing work includes a detailed assessment of the quality of the heuristics, given the shape of the series-parallel graph. This requires conducting more comprehensive experiments, with randomly generated graphs of particular shape (elevation, number of edges, node degree, and so on). We also hope to succeed in simplifying the integer linear program for some problem instances, thereby providing an absolute measure of the quality of the various heuristics. Finally, our future research will investigate general mappings, and assess the difference with DAG-partition mappings, both from a theoretical and a practical perspective. 


\section{References}

[1] M. Al Faruque, R. Krist, and J. Henkel. ADAM: Run-time agent-based distributed application mapping for on-chip communication. In Proc. of DAC'08, the 45th Design Automation Conf., pages 760-765, June 2008.

[2] AMD. ACP - The Truth About Power Consumption Starts Here. http: //www.amd.com/us/Documents/ 43761C_ACP_WP_EE.pdf, 2010.

[3] G. Ascia, V. Catania, and M. Palesi. Multi-objective mapping for meshbased NoC architectures. In Proc. of CODES+ISSS'04, the Int. Conf. on Hardware/ Software Codesign and System Synthesis, pages 182-187, Sept. 2004.

[4] N. Bansal, T. Kimbrel, and K. Pruhs. Speed scaling to manage energy and temperature. Journal of the ACM, 54(1):1-39, 2007.

[5] A. Benoit, P. Renaud-Goud, and Y. Robert. Performance and energy optimization of concurrent pipelined applications. In Proc. of IPDPS, the Int. Parallel and Distributed Processing Symp. IEEE CS Press, May 2010.

[6] A. Benoit and Y. Robert. Mapping pipeline skeletons onto heterogeneous platforms. Journal of Parallel and Distributed Computing (JPDC), 68(6):790-808, 2008.

[7] P. Blaha, K. Schwarz, G. Madsen, D. Kvasnicka, and J. Luitz. WIEN2k: An Augmented Plane Wave Plus Local Orbitals Program for Calculating Crystal Properties - User's guide, 2001. Vienna Univ. of Technology, Austria.

[8] G. Blake, R. Dreslinski, and T. Mudge. A survey of multicore processors. Signal Processing Magazine, 26(6):26-37, Nov. 2009.

[9] G. Chen, F. Li, M. Kandemir, and M. J. Irwin. Reducing NoC energy consumption through compiler-directed channel voltage scaling. SIGPLAN Not., 41:193-203, 2006.

[10] G. Chen, F. Li, S. Son, and M. Kandemir. Application mapping for chip multiprocessors. In Proc. of DAC'08, the 45th Design Automation Conf., pages 620-625, June 2008.

[11] J.-J. Chen. Expected energy consumption minimization in DVS systems with discrete frequencies. In Proc. of SAC'08, Symp. on Applied Computing, pages 1720-1725, 2008.

[12] J.-J. Chen and C.-F. Kuo. Energy-Efficient Scheduling for Real-Time Systems on Dynamic Voltage Scaling (DVS) Platforms. In Proc. of the Int. Workshop on Real-Time Computing Systems and Applications, pages 28$38,2007$.

[13] J.-J. Chen and T.-W. Kuo. Procrastination determination for periodic realtime tasks in leakage-aware dynamic voltage scaling systems. In Proc. of ICCAD'07, the Int. Conf. on Computer-aided design, pages 289-294, 2007. 
[14] Z. Chishti, M. Powell, and T. Vijaykumar. Optimizing replication, communication, and capacity allocation in CMPs. In Proc. of ISCA'05, the 32nd Int. Symp. on Computer Architecture, pages 357-368, June 2005.

[15] Cplex. ILOG CPLEX: High-performance software for mathematical programming and optimization. http://www.ilog.com/products/cplex/.

[16] DataCutter. DataCutter Project: Middleware for Filtering Large Archival Scientific Datasets in a Grid Environment. http://www.cs.umd.edu/ projects/hpsl/ResearchAreas/DataCutter.htm.

[17] M. R. Garey and D. S. Johnson. Computers and Intractability; A Guide to the Theory of NP-Completeness. W. H. Freeman \& Co., New York, NY, USA, 1990.

[18] R. Ge, X. Feng, and K. W. Cameron. Performance- constrained distributed DVS scheduling for scientific applications on power-aware clusters. In Proc. of the Conf. on SuperComputing (SC), page 34. IEEE CS, 2005.

[19] P. Grosse, Y. Durand, and P. Feautrier. Methods for power optimization in SOC-based data flow systems. ACM Trans. Des. Autom. Electron. Syst., 14:38:1-20, June 2009.

[20] Y. Gu and Q. Wu. Maximizing workflow throughput for streaming applications in distributed environments. In Proc. of ICCCN'10, the Int. Conf. on Computer Communication Networks. IEEE CS, 2010.

[21] M. Hammoud, S. Cho, and R. Melhem. ACM: An Efficient Approach for Managing Shared Caches in Chip Multi- processors. In Proc. of HiPEAC'09, the 4th Int. Conf. on High Performance Embedded Architectures and Compilers, pages 355-372, 2009.

[22] M. Hammoud, S. Cho, and R. Melhem. A dynamic pressure-aware associative placement strategy for large scale chip multiprocessors. Computer Architecture Letters, 9(1):29-32, Jan. 2010.

[23] Intel XScale technology. http://www.intel.com/design/intelxscale.

[24] J. Jaehyuk Huh, C. Changkyu Kim, H. Shafi, L. Lixin Zhang, D. Burger, and S. Keckler. A NUCA Substrate for Flexible CMP Cache Sharing. IEEE Trans. on Parallel and Distributed Systems, 18(8):1028-1040, 2007.

[25] W. Jang and D. Pan. A3MAP: Architecture-Aware Analytic Mapping for Networks-on-Chip. In Proc. of DAC'10, the 15th Asia and South Pacific Design Automation Conference, pages 523-528, Jan. 2010.

[26] R. Jejurikar, C. Pereira, and R. Gupta. Leakage aware dynamic voltage scaling for real-time embedded systems. In Proc. of $D A C^{\prime} 04$, the 41 st annual Design Automation Conference, pages 275-280, 2004.

[27] C. Kim, D. Burger, and S. W. Keckler. An adaptive, non- uniform cache structure for wire-delay dominated on-chip caches. SIGOPS Oper. Syst. Rev., 36:211-222, Oct. 2002. 
[28] K. H. Kim, R. Buyya, and J. Kim. Power Aware Scheduling of Bag-of-Tasks Applications with Deadline Constraints on DVS-enabled Clusters. In Proc. of CCGRID 2007, the 7th IEEE Int. Symp. on Cluster Computing and the Grid, pages 541-548, May 2007.

[29] K. Lahiri, A. Raghunathan, S. Dey, and D. Panigrahi. Battery-driven system design: a new frontier in low power design. In Proc. of $D A C^{\prime} 02$, the 7th Design Automation Conference and the 15th Int. Conf. on VLSI Design, pages 261-267, 2002.

[30] P. Langen and B. Juurlink. Leakage-aware multiprocessor scheduling. $J$. Signal Process. Syst., 57(1):73-88, 2009.

[31] S. Lee and T. Sakurai. Run-time voltage hopping for low-power real-time systems. In Proc. of DAC'00, the 37th Design Automation Conference, pages 806-809, 2000.

[32] P. Mahr, C. Lorchner, H. Ishebabi, and C. Bobda. SoC- MPI: A Flexible Message Passing Library for Multiprocessor Systems-on-Chips. In Proc. of ReConFig'08, the Int. Conf. on Reconfigurable Computing and FPGAs, pages 187-192, Dec. 2008.

[33] R. McClatchey, F. Estrella, J.-M. Le Goff, Z. Kovacs, and N. Baker. Object databases in a distributed scientific workflow application. In Proc. of BIWIT'97, the 3rd Basque Int. Workshop on Information Technology, pages 11-21, July 1997.

[34] M. P. Mills. The internet begins with coal. Environment and Climate News, 1999.

[35] A. Naveh, E. Rotem, A. Mendelson, S. Gochman, R. Chabukswar, K. Krishnan, and A. Kumar. Power and Thermal Management in the Intel CoreTM Duo Processor. Intel Technology Journal, 10(2):109-122, May 2006.

[36] L. Niu. Energy Efficient Scheduling for Real-Time Embedded Systems with QoS Guarantee. In Proc. of RTCSA, the 16th Int. Conf. on Embedded and Real-Time Computing Systems and App., pages 163 -172, Aug. 2010.

[37] T. Okuma, H. Yasuura, and T. Ishihara. Software energy reduction techniques for variable-voltage processors. Design Test of Computers, IEEE, 18(2):31-41, Mar. 2001.

[38] K. Olukotun, B. A. Nayfeh, L. Hammond, K. Wilson, and K. Chang. The case for a single-chip multiprocessor. SIGPLAN Not., 31:2-11, Sept. 1996.

[39] J. D. Owens, W. J. Dally, R. Ho, D. N. J. Jayasimha, S. W. Keckler, and L.S. Peh. Research Challenges for On-Chip Interconnection Networks. IEEE Micro, 27:96-108, 2007.

[40] R. B. Prathipati. Energy efficient scheduling techniques for real-time embedded systems. Master's thesis, Texas A\&M University, May 2004.

[41] J. Qin and T. Fahringer. Advanced data flow support for scientific grid workflow applications. In Proc. of $\mathrm{SC}^{\prime} 07$, the Conf. on Supercomputing, pages $1-12$, Nov. 2007. 
[42] P. Renaud-Goud. Source Code for the Experiments. http://graal. ens-lyon.fr/ prenaud/sp-cmp/.

[43] F. Schueller, J. Qin, F. Nadeem, R. Prodan, T. Fahringer, and G. Mayr. Performance, Scalability and Quality of the Meteorological Grid Workflow MeteoAG. In Proc. of 2nd Austrian Grid Symp., Univ. Innsbruck, Sept. 2006.

[44] L. Silva, G. Granato, A. Bressan, C. Lacey, C. Baugh, S. Cole, and C. Frenk. Modelling dust in galactic seds: Application to semi-analytical galaxy formation models. Astrophysics and Space Science, 276:1073-1078, 2001.

[45] StreamIt Project. http://groups.csail.mit.edu/cag/streamit/apps/ stream-graphs.

[46] J. Subhlok and G. Vondran. Optimal mapping of sequences of data parallel tasks. In Proc. of PPoPP'95, the 5th Symp. on Principles and Practice of Parallel Programming, 1995.

[47] J. Subhlok and G. Vondran. Optimal latency-throughput tradeoffs for data parallel pipelines. In Proc. of SPAA, the Symp. on Parallelism in Algorithms and Archi., 1996.

[48] L. Wang, G. von Laszewski, J. Dayal, and F. Wang. Towards Energy Aware Scheduling for Precedence Constrained Parallel Tasks in a Cluster with DVFS. In Proc. of CCGrid'2010, the 10th Int. Conf. on Cluster, Cloud and Grid Computing, pages 368-377, May 2010.

[49] R. Xu, R. Melhem, and D. Mossé. Energy-aware scheduling for streaming applications on chip multiprocessors. In Proc. of RTSS'07, the 28th IEEE Int. Real-Time Systems Symp., pages 25-38, 2007.

[50] R. Xu, D. Mossé, and R. Melhem. Minimizing expected energy consumption in real-time systems through dynamic voltage scaling. ACM Trans. Comput. Syst., 25(4):9, 2007.

[51] L. Yang and L. Man. On-Line and Off-Line DVS for Fixed Priority with Preemption Threshold Scheduling. In Proc. of ICESS'09, the Int. Conf. on Embedded Software and Systems, pages 273-280, May 2009.

[52] Y. Zhao, M. Wilde, I. Foster, J. Voeckler, T. Jordan, E. Quigg, and J. Dobson. Grid middleware services for virtual data discovery, composition, and integration. In Proc. of MGC'04, the 2nd workshop on Middleware for Grid Computing, pages 57-62. ACM, 2004. 
Centre de recherche INRIA Grenoble - Rhône-Alpes 655, avenue de l'Europe - 38334 Montbonnot Saint-Ismier (France)

Centre de recherche INRIA Bordeaux - Sud Ouest : Domaine Universitaire - 351, cours de la Libération - 33405 Talence Cedex Centre de recherche INRIA Lille - Nord Europe : Parc Scientifique de la Haute Borne - 40, avenue Halley - 59650 Villeneuve d'Ascq Centre de recherche INRIA Nancy - Grand Est : LORIA, Technopôle de Nancy-Brabois - Campus scientifique 615, rue du Jardin Botanique - BP 101 - 54602 Villers-lès-Nancy Cedex

Centre de recherche INRIA Paris - Rocquencourt : Domaine de Voluceau - Rocquencourt - BP 105 - 78153 Le Chesnay Cedex

Centre de recherche INRIA Rennes - Bretagne Atlantique : IRISA, Campus universitaire de Beaulieu - 35042 Rennes Cedex Centre de recherche INRIA Saclay - Île-de-France : Parc Orsay Université - ZAC des Vignes : 4, rue Jacques Monod - 91893 Orsay Cedex

Centre de recherche INRIA Sophia Antipolis - Méditerranée : 2004, route des Lucioles - BP 93 - 06902 Sophia Antipolis Cedex 